\title{
RTAP Evaluation
}

K. Cupps
S. Elko
P. Folta
J. Byrd
P. Kale
J. Laycak

K. Cupps

S. Elko

P. Folta

P. Kale

J. Laycak
W. Ng

V. Renbarger

R. Kyker

J. Silveira

B. Tapley

January 23, 1995

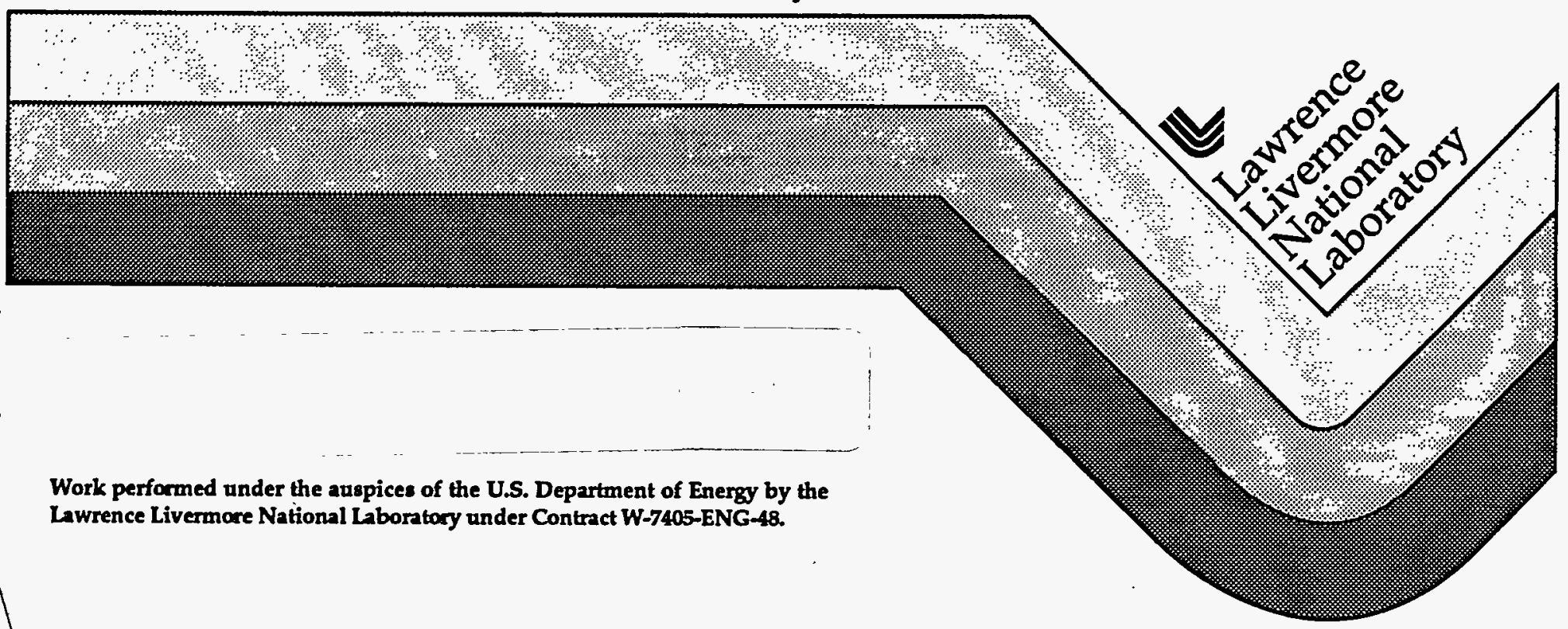




\section{DISCLAIMER}

This document was prepared as an account of work sponsored by an agency of the United States Government. Neither the United States Government nor the University of California nor any of their employees, makes any warranty, express or implied, or assumes any legal liability or responsibility for the accuracy, completeness, or usefulness of any information, apparatus, product, or process disclosed, or represents that its use would not infringe privately owned rights. Reference herein to any specific commercial product, process, or service by trade name, trademark, manufacturer, or otherwise, does not necessarily constitute or imply its endorsement, recommendation, or favoring by the United States Government or the University of California. The views and opinions of authors expressed herein do not necessarily state or reflect those of the United States Government or the University of California, and shall not be used for advertising or product endorsement purposes.

This report has been reproduced directly from the best available copy.

Available to DOE and DOE contractors from the Office of Scientific and Technical Information

P.O. Box 62, Oak Ridge, TN 37831

Prices available from (615) 576-8401, FTS 626-8401

Available to the public from the

National Technical Information Service

U.S. Department of Commerce

5285 Port Royal Rd.

Springfield, VA 22161 


\section{DISCLAIMER}

Portions of this document may be illegible in electronic image products. Images are produced from the best available original document. 


\section{RTAP Evaluation Document}

\section{Table of Contents:}

1.0 Overview

2.0 Definitions

3.0 Architectural Design

3.1 Future Considerations

4.0 Database Design and Configuration

4.1 Database Design4.2 Database Configuration

4.2.1 RtapDbConfig

4.2.1.1 Method

4.2.1.2 Advantages

4.2.1.3 Disadvantages

4.2.2 Point Configuration Files

4.2.2.1 Method

4.2.2.2 Advantages

4.2.2.3 Disadvantages

4.2.3 Shell Scripts

4.2.3.1 Method

4.2.3.2 Advantages

4.2.3.3 Disadvantages

4.2.4 Automatic Configuration

4.2.4.1 Method

4.2.4.2 Modifications to Existing Software

4.2.4.3 Advantages

4.2.4.4 Disadvantages

4.3 Future Considerations

5.0 Graphical User Interface

$5.1 \mathrm{SOM}$

5.1.1 SOM Server Launcher

5.1.1.1 Future Considerations

5.1.2 SOM Server

5.1.2.1 Future Considerations

5.1.3 Modifications to Existing Software

5.1.3.1 Future Considerations

5.2 A Comparison of RTAP and the System GUI

5.2.1 RTAP

5.2.1.1 User Interaction with RtapSchematX

5.2.1.2 Dynamics

5.2.2 System GUI

5.2.2.1 User Interaction with SOM

5.2.2.1.1 Seleting Objects to Control and Trend

5.2.2.1.2 Displaying and Printing Schematics

5.2.2.2 Dynamics 


\subsubsection{Comparison}

5.2.3.1 Features Absent in RTAP

5.2.3.1.1 Display Capabilities

5.2.3.1.2 Interaction Capabilities

5.2.3.2 Features Absent in the System GUI

5.2.3.2.1 Display Capabilities

5.2.4 Conclusion

5.2.3.2.2 Interaction Capabilities

5.3 Trending

5.3.1 Advantages

5.3.2 Disadvantages

5.3.3 Future Considerations (SCL, TCL, TCLX)

5.4 Future Considerations

6.0 Communicating with Foreign Devices

6.1 Scan Tasks

6.1.1 Heater Control Scan Task

6.2 Server Tasks

6.2.1 ODC Server Launcher

6.2.1.1 Future Considerations

6.2.2 ODC Server

6.2.2.1 Future Considerations

6.2.3 Modifications to Existing Software

6.2.3.1 ODC_FEP_SERVER Module

6.2.3.2 ODC_STRUCTURES Module

6.2.3.3 FS_COMM Module

6.3 Future Considerations

7.0 Alarming

8.0 Performance

9.0 Recommendations

Appendix A

Technical Issues

Appendix B

Development Environment

Appendix C

Utilities (data coercion, client/server) 


\subsection{Overview}

An in-depth analysis of the RTAP product was undertaken within the CNC associate program to determine the feasibility of utilizing it to replace the current Supervisory Control System that supports the AVLIS program. This document contains the results of that evaluation.

For the last three years $\mathrm{CNC}$ has evaluated many commercial control systems for possible use in the AVLIS Facility in B490 and/or an AVLIS production plant. These solutions included Distributed Control Systems (DCSs) and Supervisory Control and Data Acquisition (SCADA) systems. The results of these evaluations are documented in a report entitled "Evaluation of Commercial Control Systems".

The current supervisory control system in AVLIS must be modified to provide functional capabilities that are not currently available. These functions include the enhancement of alarm capabilities, the requirement to support additional lasers and separator vessels while maintaining current or better performance as the AVLIS system scales up, and the continued enhancement of automation in Laser and Separator systems.

With some fundamental redesign the current Supervisory Control system could meet the needs described above. The redesign would require a large amount of software rewriting and would be very time consuming. The higher level functionality (alarming, automation, etc.) would have to wait until its completion. Our current understanding and preliminary testing indicate that using commercial software is the best way to get these new features at the minimum cost to the program. Additional savings will be obtained by moving the maintenance costs of the basic control system from in-house to commercial industry and allowing our developers to concentrate on the unique control areas that require customization.

Our current operating system, VMS, has become a hindrance. Lack of available software tools, slow commercial software version updates, and dependence on a single vendor have slowed development and impaired integration. The UNIX operating system has become the choice for most scientific and engineering systems and we should follow suit.

As a result of the commercial system survey referenced above we selected RTAP, a SCADA product developed by Hewlett Packard (HP), as the most favorable product to replace the current supervisory system in AVLIS. It is an extremely open system, with a large, well defined Application Programming Interface (API). This will allow the seamless integration of unique front end devices in the laser area (e.g. Optical Device Controller). RTAP also possesses various functionality that is lacking in our current system: integrated alarming, real-time configurable database, system scalability, and a Sequence Control Language 
(SQL developed by CPU, an RTAP Channel Partner) that will facilitate the automation necessary to bring the AVLIS process to plant-line operation. It runs on HP-9000, DEC-Alpha, IBM-RS6000 and Sun Workstations.

Early investigation, planning, and training laid the ground work for the RTAP evaluation effort. The RTAP evaluation phase began in earnest in February 1994. During this phase, $10 \mathrm{CNC}$ people possessing different areas of expertise were involved to varying degrees. The evaluation required approximately 20 man months of effort. The main thrust was to prototype two AVLIS subsystems: Heater Control from the Separator system and the Dye Combination Wall from the Process Laser system. The RTAP licenses were purchased and installed on two HP machines in our development environment.

The prototype of the Heater Control subsystem included a redesign of 2 Graphical User Interface (GUI) screens and associated control panels with the RTAP equivalent (SchematX Builder, Control Panel Builder, and SchematX applications). It also included the configuration of the Programmable Logic Controller (PLC) scan task and the RTAP real-time database. The end result was the ability to send commands from the control panels and see the resulting effects in the PLC, the RTAP database, and the SchematX screens.

The prototype of the Dye Combination Wall included connecting the current System Oriented Man Machine Interface (SOM) to RTAP, configure the RTAP database, and developing an ODC auto-generate application that would allow the ODC portions of the RTAP database to be generated from the configuration files of the ODC. The end result was the ability to populate the ODC points of the database automatically and to run the SOM to monitor those points.

During the evaluation period we enlisted the help of a consultant, Marc Chevis, from CPU, Inc. Marc and his company have a great deal of experience with RTAP and have provided various products as an RTAP Channel Partner. Marc was on-site for two week-long consultation visit and provided phone-support. His help was invaluable in lowering the RTAP learning curve.

The evaluation supported RTAP's feature rich claims and we found that we were able to utilize its open architecture to integrate several of our existing systems (e.g. SOM, ODC) in a straightforward manner. As a result, we are recommending that the RTAP product be used be as the supervisory control system for the AVLIS program. The following document contains the detailed basis for that decision. 


\subsection{Architectural Design}

Many factors went into the decision process for selecting an architectural design for the evaluation implementation. The primary objective was to provide a test bed for investigating the many different characteristics associated with the RTAP system. Those characteristics that were the focal point for the architectural design include:

- Distributed Processing

- Interoperability Between Existing Applications

- Graphical User Interface

- FEP Communications

Distributed Processing: One of the inherit traits of the RTAP system is its ability to provide transparent access between remote environments. This particular trait is essential in providing a mechanism for developing a framework that is both flexible and scalable. It is for this reason that we selected two subsystems of the current supervisory system for evaluating this characteristic. The selected subsystems included a portion of the Closed Loop Heater Control and the Dye Combination Wall systems. Each of these subsystem's constituted an RTAP environment. Though multiple environments can coexist locally, we elected to implement them in a distributed fashion (Figure 3.1). This implementation would afford us the opportunity to evaluate RTAP's scalability factor as well as evaluating it's distributed capabilities between remote environments. (From a programming standpoint the TCP port numbers assigned to each of the environments are defined within the /etc/services file. The corresponding port number for the Closed Loop Heater Control environment is 22806 while the port number for the Dye Combination Wall environment is 22808.)

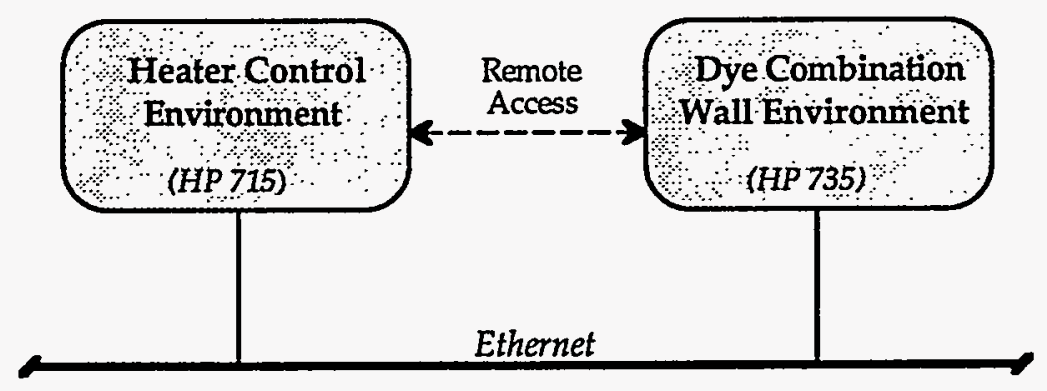

Figure 3.1 Distributed RTAP Environments

Interoperability Between Existing Applications: The objective of this investigation was to determine the feasibility of adapting existing supervisory applications into an RTAP environment. In addressing this issue we had to be cognizant of interoperability issues such as implementing an alternate networking protocol (TCP/IP vs. DECnet) as well as handling the different data representations between the HP and VAX architectures. The applications that were selected for this investigation included SOM and the ODC FEP Server applications (Figure 3.2). The SOM application was selected because it could also be used for the 
graphical user interface's investigation. Similarly, the FEP Server application was selected because it could also be used for the FEP communication investigation.

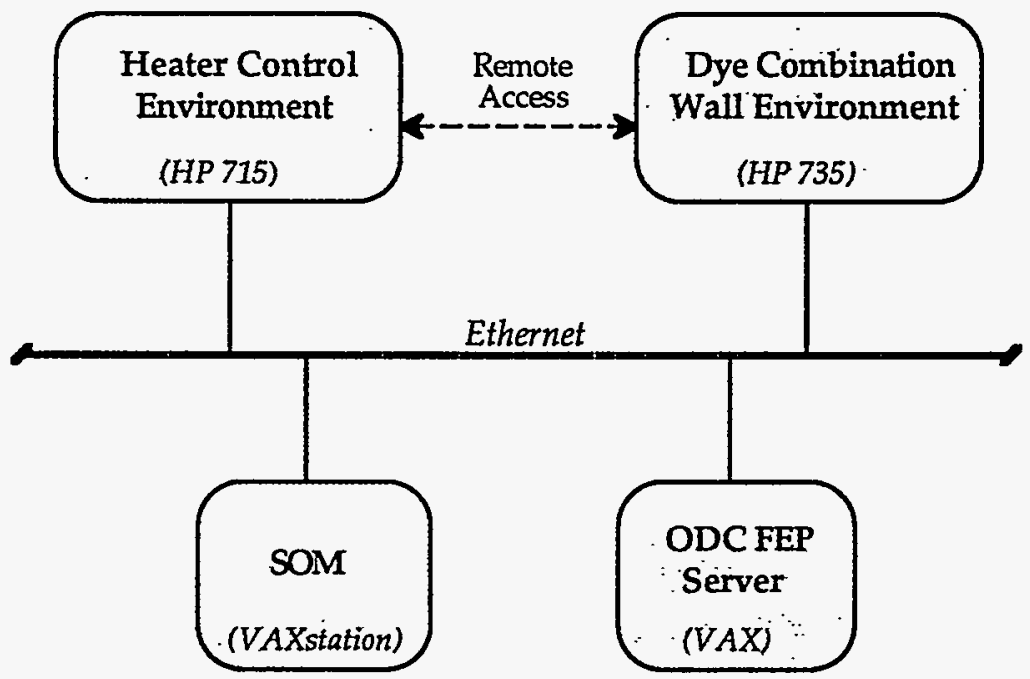

Figure 3. 2 Integrating Existing Applications into the Architectural Design

Graphical User Interface: An integral part of the RTAP base system is a graphical user interface tool called SchematX. It was the intention of this investigation to evaluate the capabilities associated with this tool. Furthermore, by integrating SOM into an RTAP environment, we can compare and contrast the SchematX tool with our existing capabilities.

FEP Communications: Within the current U-AVLIS control system, the communication mediums employed between the supervisory system and the FEPs is performed either through the RS232 or Ethernet medium. By implementing the Heater Control and the Dye Combination Wall subsystems, we will be able to evaluate each of these communication mediums within an RTAP environment.

In the current U-AVLIS supervisory system, Ethernet-based FEP communications is accomplished through an intermediate process (such as the ODC FEP Server). These processes utilizes DECnet as the networking protocol. For the evaluation implementation, we wanted to retain the intermediate process approach but needed to investigate the transition from a proprietary networking protocol to a more standard protocol. For reliability reasons, we elected to investigate the $\mathrm{TCP} / \mathrm{IP}$ networking protocol as a replacement for DECnet.

In addressing RS232 communications, we elected to investigate RTAP's scan task capabilities. An RTAP scan task accomplishes all communication functions and data type conversions between a host and a remote scan device, (such as the PLC's). RTAP supports both the development of a new scan task or the ability to customize any existing scan task. Due to time constraints, we elected to procure a commercial scan task to communicate with the Heater Control subsystem. To 
accomplish this objective required the PLC 5 system to be equipped with a special board (model 1785-KE) that would convert between RS232 and the DataHighway Plus protocols. Figure 3.3 illustrates how we designed FEP communications into the evaluation implementation.

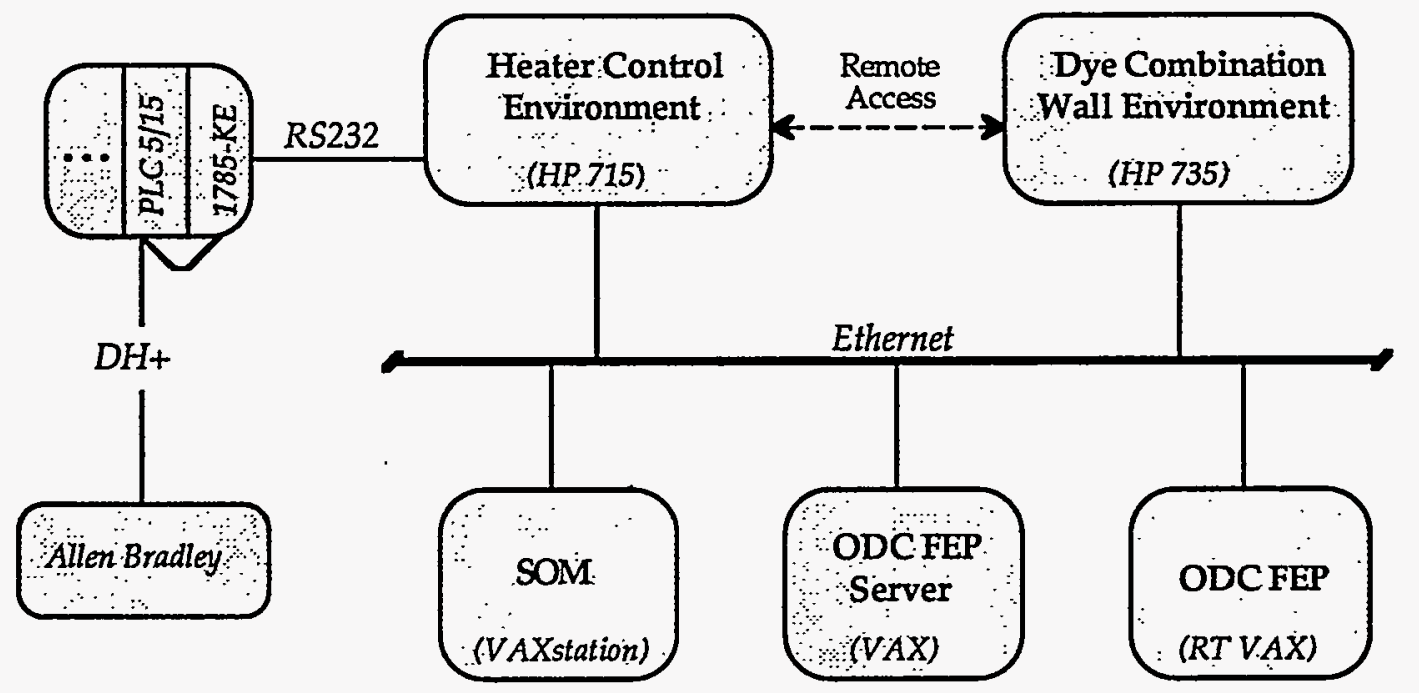

Figure 3.3 Designing FEP Communications into the Evaluation Implementation

\subsection{Future Considerations}

Dataless Environment: A dataless RTAP environment provides a mechanism for off loading computational resources from a host containing an RTAP database. Future architectures should consider the use of dataless environments for maximizing a design's scalability factor.

Calculation Engine Limitation: There exists a limitation in RTAP's Calculation Engine (CE) within a multi-environment architecture. An event in one environment cannot transparently trigger a $C E$ within another environment. To accomplish this objective would require each environment to contain related points; one being the master with the other acting as a slave point. An auxiliary application would then need to be developed to monitor the master point. When a value associated with a master point is updated, the application monitoring that point would write the updated value into the corresponding slave point's value. Once the slave's value is updated, the $C E$ associated with that slave point would be triggered. For scalability purposes, the auxiliary program can be executed in any environment including a dataless environment. 


\subsection{Database Design and Configuration}

The RTAP Database is the heart and soul of most RTAP systems, thus proper database design is crucial for a successful implementation. During the evaluation period much effort was spent configuring the Heater Control Database (HCDB) and the Dye Combination Wall Database (DCWDB). There is, obviously, a big difference between configuration and design. Database design was not examined in the depth that it deserves due to the short time frame of the evaluation, but a few useful ideas were discovered.

\subsection{Database Design}

The RTAP database is a hierarchical representation of data. It contains at least three distinct sub-trees. One sub-tree is "pointTemplates" and it is used to store a template for each kind of object that can be created in the database. An example of a point in a "pointTemplates" sub-tree is odcTemplates. The points in the odcTemplates branch include analog_input, analog_output, closed_loop, cross_couple, device, digital_input, digital_output, hbc_loop, stepping_motor, and video_input. Each of these points contains attributes applicable to only its point type. A video_input point, for example, contains 8 attributes: the $x$ and $y$ centroid positions, their desired positions, their average positions and their local error. When a new video input is added to an ODC, it is not a major undertaking to update the database. The video_input point in the odcTemplates branch is copied to the desired location in the database. One simply edits the copied point and changes the name and alias and a new video input has been created. There is no messing around creating the 8 attributes since they are copied over with the point and their names don't change. The copy process takes care of automatic generation of a unique alias for the point and all of its attributes. The system generated aliases are made up of letters and numbers and may be changed by the user to something more descriptive if desired.

Another sub-tree is "support_data" and it contains configuration data for scan tasks, alarms, the historian, etc. that are used in RTAP. The "support_data" subtree of the database currently contains branches for alarm_class, class_config, database_config, historian_config, plot_config, scan_config, schedule_config, and users_config. The DCWDB database does not currently have all of these branches.

The other sub-trees are used to represent the data values being sent from/to various external sources (GUIs, FEPs, etc.) as well as any internal or pseudo points needed in the system. For instance in the database that was created for DCWDB, there is a sub-tree for "VAAC", "ABD", and "ODC". In the database created for Separators, there is a sub-tree "POD1", with a sub-branch called "HEATER_CONTROL". Other appropriate branches to add at the same level as 
"HEATER_CONTROL" would be "FEEDER", "MAGNETS", etc. The separation of control and monitoring points (the real data) from the configuration and maintenance data ("support_data" sub-tree) is an important database design technique.

Another design aspect that must be considered is point design. Points should be separated into groups that indicate that they are all of a certain kind. For instance, if one wanted to plot all analog input values there should be something that indicates to the plotter that a specific point is an analog_input. You don't want to have to specify each analog_input by name. The mechanism used to group similar kinds of points is called a point class. By defining points via a template and assigning each template a different class number, external applications are able to perform operations on a class of points simply by specifying the point class number (any number up to 64K). Another method of grouping is categories. Categories are primarily used for security.

There are many other areas of point design that were not addressed. Alarming will affect point design as will archiving, plotting, and logging. Since alarming, archiving, plotting and logging were not part of the evaluation, none of the point design issues that relate to those areas were addressed. Much work remains in the point design area.

\subsection{Database Configuration}

Database Configuration can be done via many different methods, which in the end achieve the same effect. The various configuration methods include using RtapDbConfig, using the PConfig (point configuration) files, using shell scripts to "quicken" the PConfig process, and finally writing a program that makes calls to the RTAP Application Program Interface (API), rtConfigDatabase. We used each of these methods, to different extents, to configure either the HCDB or the DCWDB. A description of each method and its advantages and disadvantages follows.

\subsubsection{RtapDbConfig (Using Point and Click Interface)}

\subsubsection{Method:}

RtapDbConfig is an option from the RTAP tools palette. It is used to add, copy or delete points from the Database. Once the RtapDbConfig icon is selected a tree representation of the database comes up in a new window. There are several options available on the menu bar. These include Environment, Edit, View, and Options. Choosing the Edit menu item invokes a pull-down menu with menu items to Cut, Copy, Paste (branch and point), Load branch, Unload branch, Create point, Delete branch, or Edit Characteristics. 
Deleting a leaf point is quick and simple, as is deleting an entire branch. Creating a point entails giving it a name and alias, each of which can be a maximum of 19 characters; specifying whether the point is disk or RAM resident; as well as defining whether the order of Calculation Engine function execution is user defined or natural execution order. The time to create a point is not that bad, if you only have one or two to do.

The main discovery made during the evaluation of RtapDbConfig is that there is no way to create or add attributes to a point via this tool or any other RTAP point and click interface. All attributes must be defined in a PConfig file and then loaded into the database via the "Load Branch" command in the Edit menu of RtapDbConfig. This is a large omission in capability. Attributes are the only entities in the database that have values associated with them, and as such are of utmost importance. It would be nice to be able to add one or two with the RtapDbConfig tool. It would not be useful to use RtapDbConfig for more than one or two attributes since they require much more information than points require.

\subsubsection{Advantages:}

The advantage of using RtapDbConfig is that it provides a graphical view of the data hierarchy tree. One can easily see how the data is arranged. It is useful for adding a very small number of points ( 2 or 3 ) and for deleting entire branches of the database. This database manipulation can be done without taking the system down, which is a big plus for users. It means it is possible for users to add a small number of new points to the database without having to bring in the services of a computer scientist. For large scale database modifications the tool is essentially useless.

\subsubsection{Disadvantages:}

The biggest disadvantage of using RtapDbConfig is that it is extremely time consuming and tedious when adding anything other than a small number of points. There is much clicking back and forth between various sub-menus and dialog boxes, and it's just too slow. It's one of those things that you do for about five minutes before you hear a voice screaming "there must be another way!"

\subsubsection{Point Configuration Files (PConfig files)}

\subsubsection{Method:}

PConfig files are ASCII text files used to describe points. These text files must have a particular format and they can be created with any text editor. RtapDBConfig can be used to load a branch of these PConfig files (using "Load Branch" under the Edit menu) and it can also be used to unload a branch of the database (using "Unload Branch" under the Edit menu) into PConfig files. Two 
RTAP utilities also exist that use the PConfig files--RtapDbLoader and RtapDbUnloader. A whole or partial database can be loaded from PConfig files using RtapDbLoader. RtapDbUnloader can be used to unload a whole or partial database into PConfig files.

PConfig files can be created with an editor from scratch but the easiest way is to find an existing file, copy it, and then make changes to it to fit your particular point. RTAP furnishes some examples of PConfig files. A point configuration file consists of point header information, attribute specifications, and comments. Point header information and attribute specifications are made up of keywords and have values associated with them (some keywords have default values where others have to be specified). There is one point header section for each point. In this section are the fields Name (point name), Alias, Residence (RAM or Disk), Categories, CE Indicator (enabled or disabled), CE Order (natural or userdefined), and Class.

Attribute specifications are set off using the keywords BEGIN ATTRIBUTE and END. Everything within this begin/end pair is used to define the attribute. Fields are Name (attribute name), De Type (data element type, e.g. rtFLOAT), Write Groups and Read Groups (masks specifying the user group(s) that can write and read this attribute, Type (scalar, vector, or table), Value (can be used to initialize a scalar attribute), and Definition (a Calculation Engine definition). A point can have up to 255 attributes, each one having its own begin/end pair.

\subsubsection{Advantages:}

The advantage of the PConfig files is that they can be copied and edited with a text editor and it is the way to add attributes to your points. RtapDbConfig's Edit menu is used to load these files into the database.

\subsubsection{Disadvantages:}

This method is tedious if there are a lot of similar looking points. But using this method coupled with shell scripts is quite useful. Storing the whole database as PConfig files on disk can take up a lot of disk space (although seeing the hierarchical file structure is nice because it is an exact copy of what the database tree looks like).

\subsubsection{Shell Scripts (and PConfig Files)}

\subsubsection{Method:}

A shell script was designed that made the creation of similar points more automated. This shell script took three parameters: "point name", "scan table index", and "template name". "Point name" was used for the point's name, the alias, and the PConfig filename. A point that is read from the PLC via a scan task 
must have an entry in a scan input table called a scan link (for a more detailed description, see section 6.1 Scan Tasks). A point that is written to the PLC via a scan task must have an entry in the scan output table. "Scan table index" is the index (row) of the appropriate table. "Template name" is the name of the template that describes the particular kind of point. There were monitor point templates and control point templates. Within these categories, there were templates based on data type, such as rtInt16 and rtFloat.

The shell script would make the directory for the new point and do a "cd" to that directory. Then it would make a copy of the template file in that directory and name the new PConfig file "point name". Using the stream editor (sed), "point name" was substituted for POINT_NAME in the PConfig file and "scan table index" was substituted for ScanTableIndex.

This shell script was named "mknpts" (make new points). Another shell script was created to make multiple calls to "mknpts" with different parameters in order to have several PConfig files created at once. These files were loaded into the database using the Edit menu of RtapDbConfig.

\subsubsection{Advantages:}

Files could be created much more rapidly with this method as many files were alike except for point name and scan table index number. Obviously the original templates and a file with the appropriate point names and scan table indices still needed to be created. These would always have to be created somehow by a human (no matter how much automation was involved).

\subsubsection{Disadvantages:}

Using Load Branch under the Edit menu of RtapDbConfig, we never figured out a way to load a partial branch (only whole branches) but maybe it can be done. This was a hindrance when there were only a few points to add to the existing points in the HEATER_CONTROL branch. The entire HEATER_CONTROL branch had to be deleted and then reloaded (including the new points) from the PConfig files.

\subsubsection{Automatic Configuration (using calls to RTAP API)}

\subsubsection{Method:}

During the evaluation a method was developed to "automatically" populate the Optical Device Controller (ODC) portion of the DCWDB. There are several steps in generating this branch of the database. The first step is to be able to generate an ASCII (or other) file that describes all possible point classes a certain FEP handles. This is necessary so that point templates can be created and added to the "support data" branch of the database. 
In the case of the ODC there are 10 different kinds of points, as mentioned earlier. The description of these 10 point classes is contained in a "point templates" file. The file contains the following information for each point template or point class: the name of the point, the number of attributes the point has and the point class number. If a point has attributes, then each attribute is specified following the point in the file. For each attribute, the name and type (integer, float, ...) of the attribute are specified.

The program that generates the point templates file runs on the VAX and gets its data from the ODC Configuration Editor (see section 4.2.4.2 below).

The program that reads the point templates file and creates the point templates in the RTAP database is called CreateodcTemplates. The CreateodcTemplates program is called by the ODC Server whenever there is a need to completely respecify all of the templates belonging to the ODC in an RTAP database. This will usually only happen when a new version of the ODC is released, and is therefore not a common occurrence.

Once point templates have been put into the database any of these templates may be used to create a new point. The program used to configure all of the ODC points in the DCW database is called CreateODCDatabase. This program is invoked by the ODC Server when a CONFIGURE message is received from the ODC FEP Server.

CreateODCDatabase reads a points file to configure the points in the DCWDB. This points file is created by the ODC Configuration Editor and copied (FTP'd) from the VAX to an agreed upon directory on the HP. This file is assumed to exist when the CONFIGURE message is received by the ODC Server. The points file indicates new point names and aliases, how the points are hierarchically related and which templates to use to create the new points.

The CreateODCDatabase program makes a call to lock the database then copies desired point template under the specified parent point. It is at this time that the point is given its new name. Once the new point has been created and named a call is made to configure the alias. After completing the copy and configure alias process for each point in the points file the ODC portion of the DCWDB is completely configured. CreateODCDatabase unlocks the database and exits.

\subsubsection{Modifications to Existing Software}

The program that generates the point templates file for an ODC RTAP database is the ODC Configuration Editor. Modifications were made to the editor to generate this file. The extent of the modifications was very simplistic and basically involved reusing the existing code that generates the State Tree ODC macro definition file. The source of the information in the RDB database is the 
same, only the format of the output file is different. The format of the output file is describe in the following section.

\section{ODC RTAP Template File}

The purpose of this file is to define RTAP database template points to be used in the building of an RTAP database for an ODC FEP. The templates will NOT fully describe what a point looks like in the ODC database and will only contain those attributes that have been configured for storage in the RTAP database by the ODC configuration editor. The template file is regenerated upon every build of ODC parameter file but this does not imply that the contents of the template file has changed. The contents of the template file will only change when the end user requests additional information from an ODC be placed in the RTAP database.

For an ODC the following template point types will be created.

$\begin{array}{lc}\text { Name } & \text { Class type } \\ \text { ANALOG_INPUT } & 5 \\ \text { ANALOG_OUTPUT } & 9 \\ \text { CLOSED_LOOP } & 3 \\ \text { CROSS_COUPLE } & 2 \\ \text { DEVICE } & 1 \\ \text { DIGITAL_INPUT } & 7 \\ \text { DIGITAL_OUTPUT } & 10 \\ \text { HBC_LOOP } & 4 \\ \text { STEPPING_MOTOR } & 8 \\ \text { VIDEO_INPUT } & 6\end{array}$

The specified class types were assigned here only for completeness. The values are by no means locked in and in all likelyhood will change as the developement process progresses.

The file will be in ASCII format and consists of lines that provide point information and attribute information. The first line in the file will be a point line and formatted as follows

"19 character point name" "class type" "number of attributes"

The attribute lines will follow point line, the number of which are contained in the point line information, format follows

"19 character attribute name" "De Type" "Point Type"

\subsubsection{Advantages:}


This method has many advantages over manually configuring the database. The first and foremost is that it is much faster and requires no human effort once the protocol is established (assuming no errors, of course). Another advantage is that possibility of data entry error is eliminated. Since the FEP is generating the data one can be sure the FEP and Supervisory system agree on what points are in the system. The reason to automate is to end long hours of tedious data entry for an entire database. The automated configuration method described above accomplishes elimination of tedious data entry.

\subsubsection{Disadvantages:}

One of the main disadvantages is that no method for simply adding one or two points to the database has been established as part of the messaging protocol. The other disadvantage is that when an error is encountered in the program (say, because of unknown resource constraints), it will require a programmer to dig around in a log file to figure out what went wrong. The users will not be involved in the creation of the database so they won't understand the process or the symptoms of having a problem occur. They probably won't notice the problem until they are trying to do something on some completely unrelated screen and there's a read/write lock on the database. Then we will get a call about frozen data values and nothing is working... This is always the price of automation. Errors must be minimal and error handling must be excellent or users will end up very frustrated.

\subsection{Future Considerations}

Future considerations include investigation of third party database configuration tools, further enhancement of automated database configuration capabilities as well as many unanswered questions about point design.

Database configuration tools available from tesserNet include a Database Builder and a Database Point Creator. The Database Point Creator sounds much like the CreatteodcTemplates program described above, but may have a GUI built on top for easy definition. The Database Builder sounds particularly attractive as it provides full add, delete, copy and move functions of points as well as grouping of attributes with multiple attribute groups in each point, if desired. tesserNet is willing to ship a demo copy of both of these products.

Automated database configuration capabilities need to be expanded to meet the needs of all our FEPs. This means writing programs that will do automatic configuration of the ABD, PLC and embedded controller branches of the database. Expansion also implies that we need to be able to do more than just configure the entire database for a FEP. We must be able to add, delete, and copy small numbers of new points in just as automated a fashion. 
As was mentioned earlier in the database design section, a lot of thought needs to go into the database design as well as into point classes. At this time, we don't have a good feel for how the archiver, alarms, the trender, etc. will interact with the database. We need to know how theses tasks need the database to be constructed just so everything can work properly as well as database design for speed of these tasks. Speed for all (FEPs, archiver, alarms, etc.) may turn out to be mutually exclusive. We may have to decide on a database design that allows speed for the most important tasks and the other tasks will run more slowly. Overusing the Calculation Engine (CE) is a concern. The $C E$ is somewhat expensive in time usage but we don't have a good feel for how much and what are the alternatives to using it. Point dependencies that trigger the Calculation Engine need to be seriously considered as the dependencies are point based (not attribute based). Database size and its impact on performance is another large concern. When one starts adding lots of alarm points, plot points, etc. the database can explode. Do we just split our databases up onto more machines if it is a performance problem? Point classes and how we want to group things for efficient API access is also an issue. CPU's Marc Chevis commented that it would have been nice to have had a point class for "heater control" control points to send control points to the PLC using SCL. Point design is a big area and requires much consideration in the future. 


\subsection{Graphical User Interface}

The focus of this effort was to investigate the graphical user interface options for monitoring, controlling, and trending values within an RTAP environment.

\subsection{RTAP/SOM Integration}

The purpose of integrating the SOM application into an RTAP environment was two-fold; to address interoperability issues between the VAX and the HP architectures and to compare and contrast RTAP with the System GUI. In supporting the SOM integration effort, two auxiliary programs.were developed. These auxiliary programs include the SOM Server Launcher and the SOM Server and provide the necessary communications interface between RTAP and SOM.

\subsubsection{SOM Server Launcher}

Similar with the technique used in the existing system, it was decided that each SOM would have a corresponding remote application for servicing the network communications. To minimize complexity, it was decided that all SOM applications would establish a communication link through a single "wellknown" communications address. The application that monitors this "well known" address, and acts as a gateway between SOM and RTAP, is the SOM Server Launcher.

The purpose of the SOM Server Launcher is to continually monitor the "well known" address for the arrival of a network connection request. Once a connection request arrives, the SOM Server Launcher is asynchronously notified and will attempt to accept the incoming connection. Once the communication link has been successfully established, SOM will forward a "connect" message to the SOM Server Launcher. The result of a "connect" message is that a counterpart application will be started and will be responsible for servicing the exchange of information between SOM and RTAP. To accomplish this methodology, the "connect" message must contain information regarding the SOM's host and the target RTAP environment to connect to. (One would assume that an RTAP environment name is not necessary since the SOM Server Launcher is already associated with an RTAP environment. However, by providing an environment name, SOM can establish a connection to an environment through an intermediate environment (i.e., a dataless environment) thereby exploiting RTAP's scalability trait.) This information is forwarded onto the counterpart application for re-establishing communications back to SOM. After the counterpart application begins execution, the SOM Server Launcher will return to an asynchronously state awaiting the arrival of another connection request on the "well known" communications address.

An RTAP host intending to service SOM applications must have a single SOM Server Launcher program operating within an RTAP environment. Moreover, only a single SOM Server Launcher can be executing on a given host at any one time. The SOM Server Launcher was designed to begin execution during environment 
startup and operate continuously until the environment is shutdown. This is accomplished by modifying the RTAP environment table to include the following definitions for the corresponding columns.

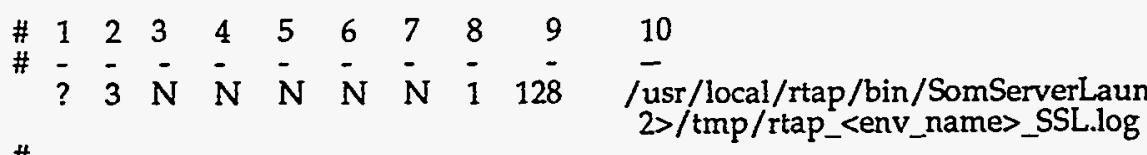

Figure 3.1 illustrates the algorithm used by the SOM Server Launcher for monitoring and accepting connections on the "well known" address. The algorithm can handle up to five simultaneous connection requests.

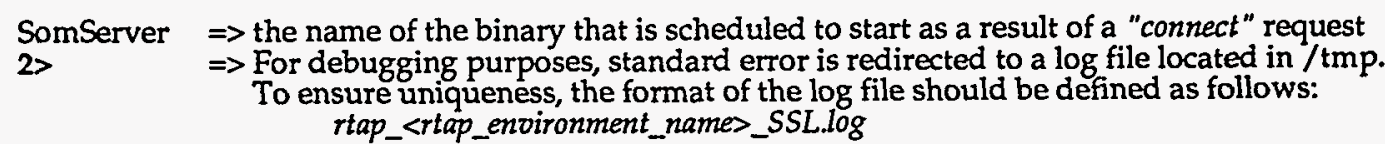

$(0, \operatorname{not} 0)$

(No, Environment, Program)

(No, Yes)

(No, Yes, Abnormal)

(No, E,L,M,P,R,W, Yes)

(0-32767)

$(0-127,128)$

\# The command line arguments are defined as follows:

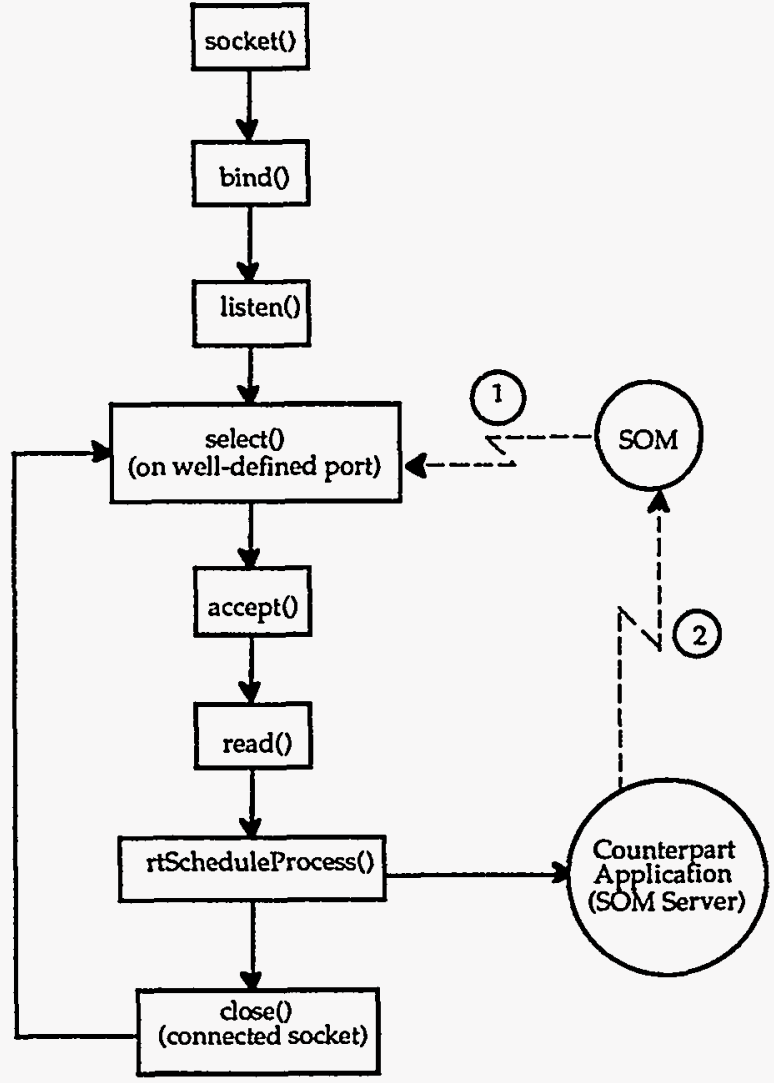

Figure 3.1 SOM Server Launcher Algorithm 
Figure 3.2 illustrates how SOMs establish a communication path to the SOM Server Launcher. A SOM Server Launcher must be running before any SOM's can establish a connection to an RTAP environment. The diagram depicts the SOM Server Launcher monitoring the "well known" address for any incoming connection requests. From a programming standpoint, the "well known" address is based on the TCP protocol with a port number of 10001 . The port number is defined within the application itself and is true for all hosts running a SOM Server Launcher.

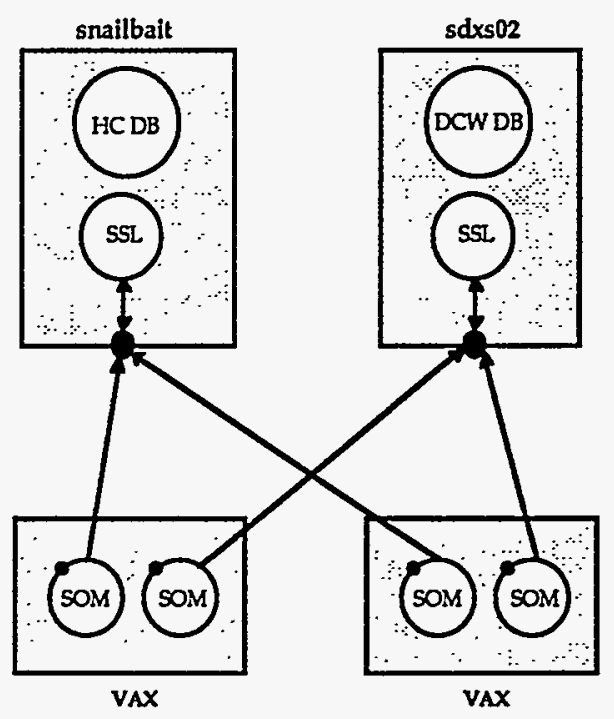

Figure 3.2 Establishing Communications Through a "Well Known" Address

\subsubsection{Future Considerations}

It was decided that the same technique employed by the SOM Server Launcher would be used for establishing communications between the ODC FEP Server and RTAP. Though an ODC Server Launcher program was developed, the only difference between it and the SOM Server Launcher is the counterpart application that is started as a result of a "connect" request. A future consideration should be to create a generic Server Launcher program that can support not only the ODC FEP Server and SOM but any future applications as well. The modifications that would need to be incorporated include:

- Adding a field within the connect message that designates what type of application initiated the request (i.e., SOM, ODC FEP Server, etc.)

- Based on the new field, start the appropriate Server application

- Modify the ODC FEP Server to use the same "well known" address as SOM 


\subsubsection{SOM Server}

The counterpart application initiated as a result of a "connect" message is the SOM Server application. The primary responsibility of the SOM Server is to marshal information between RTAP and a remote SOM application. This is accomplished by monitoring both the RTAP message queue file descriptor and a network socket descriptor. Outgoing messages are normally triggered by an RTAP message queue file descriptor while incoming messages are triggered by a socket descriptor.

Applications connected to an RTAP environment are assigned a unique RTAP message queue. RTAP message queues are used for passing interprocess information between RTAP and an application connected to an RTAP environment. Moreover, RTAP provides the ability for applications to register events and be notified when the event occurs. When an event does occurs, the RTAP message queue file descriptor is triggered indicating the message queue contains information regarding the event. Within the evaluation implementation, an RTAP event would normally indicate a change in value occurred. The updated value would then be forwarded onto the remote SOM application to be displayed.

Remote communication between an RTAP host and a SOM host were implemented using Berkeley sockets. Sockets provide the ability to perform bidirectional communication between two hosts. Prior to sending data from an RTAP host to a SOM host, the SOM Server had to perform the necessary conversions on integer and floating point data representations. This conversion must also be done when data is sent from SOM to RTAP. Refer to Appendix C for additional details regarding the data coercion process.

Outgoing information is normally triggered by an RTAP event while incoming information is triggered by the socket "select" call. Furthermore, outgoing information is extracted from the RTAP message queue and formatted into a well-defined network protocol developed for the prototype implementation. Similarly, incoming information is extracted from the network protocol and formatted into an RTAP message.

The sequence of events that must transpire for establishing communications between RTAP and SOM are highlighted below.

1) The RTAP environment that SOM intends to connect to must be started. (Note: the startup sequence implicitly starts a SOM Server Launcher.)

2) Begin a SOM session. During initialization, SOM establishes a connection to the SOM Server Launcher's well known TCP port number.

3) The SOM's host name is derived as well as an available TCP port number in which the SOM Server will re-establish communications back to SOM.

4) SOM composes a connect message that includes the derived host name, port number, and the intended RTAP environment name that SOM intends to connect to.

5) SOM sends the connect request to the SOM Server Launcher and waits for the SOM Server to re-establish communications. 
6) The SOM Server Launcher is notified that information is contained within the well known address.

7) The SOM Server Launcher reads the connect request, parses the messages and begins a SOM Server application. The SOM's host name, port number, and RTAP environment name are passed to the SOM Server.

8) After scheduling the SOM Server to begin execution, the SOM Server Launcher closes the associated socket, and waits on the well known address for another connect request.

9) The SOM Server re-establishes communications back to SOM.

10) Once a communications channel is re-established, SOM closes the connection to the SOM Server Launcher's "well known" address and communications between RTAP and SOM can begin.

Figure 3.2 depicts the resultant communication links from performing the above steps.

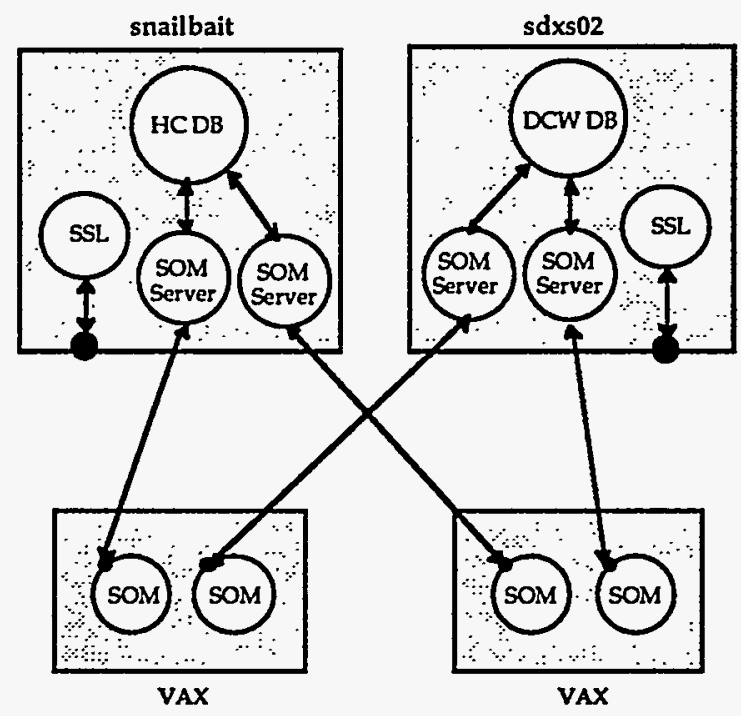

Figure 3.3 Communication Links Between RTAP and SOM

When the SOM Server is scheduled to begin execution, standard error is redirected to a log file located in / tmp. To ensure uniqueness, the format of the $\log$ file should be defined as follows:

rtap_<rtap_environment_name>_SS.<SOM_host_name>:<SOM_port_number>

The following describes the different message that are initiated from SOM and serviced by the SOM Server. They include "declare interest", "cancel interest" and a "write control point" message.

Declare Interest: Upon receiving a "declare interest" message, the SOM Server will register an RTAP database event for the point specified within the message. RTAP will in turn notify the SOM Server when a database event occurs for the specified point. Within the call to rtAttachDbEvent, the ASCII point name is placed within the handler message field. This methodology was used so that when RTAP signals that a database event occurred, the associated ASCII point name can be easily obtained through the handler message. In addition, a link list is used to retain the associate event ID for the registered event. This information 
is needed when canceling interest in a point. In addition, the associate "plin" and "ain" are queried from the database and stored in the linked list. Subsequent database transactions can use the "plin" and "ain" for direct access.

Cancel Interest: When SOM is no longer interested in a point, a "cancel interest" message is delivered to the SOM Server. The SOM Server will search the linkedlist for the ASCII point name, extract the associated event ID and make a call to rtDetachFromEvent to disassociate the event.

\section{Write Control Point:}

From SOM's perspective, points that are only displayed on a SOM screen are termed "monitor points" while points that can be displayed and changed from a SOM screen are termed "control points". In the evaluation implementation, the Heater Control environment was the only environment containing control points. When a user enters in a new "control point" value from a SOM screen, a "write control point" message is forwarded onto the SOM Server. Upon receipt, the SOM Server will write the updated value into the database and write a value of 1 into the control point's "trigger" attribute. Each "control point" has an associated "trigger" attribute that is monitored by the DataHighway Plus scan task. These "trigger" attributes are used to signal the scan task that the associated "control point" value has changed. Once the scan task is notified that a "control point" value has changed, the scan task will read and forward the updated "control point" value onto the FEP.

Database events delivered to the SOM Server indicates that a value has changed and the new value needs to be reflected on the SOM screen. The following describes the message that is initiated by an RTAP database event.

Event Notification: As previously stated, a "declare interest" message causes the SOM Server to register an RTAP database event. When the value changes, RTAP will trigger an event and forward the event onto the SOM Server. In turn, the SOM Server will extract the point name from the handler message and compose a message that will notify SOM about the changed value.

Scalability is one of the inherit traits of the RTAP system. With this in mind, the evaluation implementation successfully demonstrated the use of an intermediate RTAP environment for establishing connection to a remote RTAP environment. Figure 3.4 illustrates this scalability concept. 


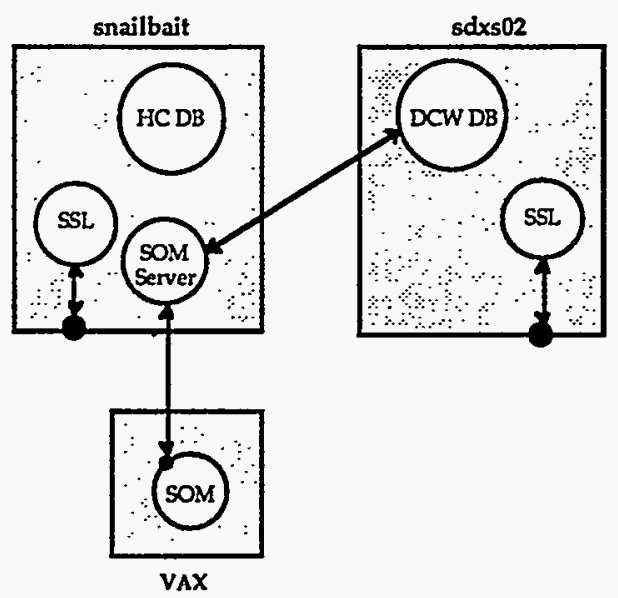

Figure 3.4 Demonstrating RTAP's Scalability Trait

\subsubsection{Future Considerations}

Message queues are the basis of the RTAP communication facility. RTAP uses message queues to perform interprocess communications and is extended to allow the exchange of messages between processes in a remote environment. Because message queues are a limited resource, there exists the potential for exhausting this resource. Moreover, since the SOM Server process is event driven, there exists a high probability that several simultaneous database value changes could exhaust this resource. Therefore, it is suggested that the SOM Server process be changed from an event driven to a polling implementation. To accomplish this objective, it is suggested that the SOM Server process use the RTAP time keeper to send a cyclic alarm clock message to the SOM Server. Upon notification, the SOM Server would perform a single RTAP read operation on all points that it is monitoring within a specific environment. The SOM Server could either forward all data values onto SOM or implement logic to filter only those data values that have changed. (Note: The polling interval should be contained within an RTAP point. This would allow the polling interval to be easily changed. The SOM Server would register a database event on the interval and when the interval is changed, the SOM Server would be asynchronously notified. Upon notification, the SOM Server could cancel the current cyclic alarm and set a new alarm based on the updated polling interval. This is an improvement over the current system. Currently, the polling interval is only changed after the next scheduled interval (i.e., if the current interval is set to 59 seconds and the interval is changed to 1 second, it could take up to 59 minutes before the 1 second interval goes into effect).)

The current implementation of the SOM Server is limited in the sense that it can only interact with points defined in a single RTAP environment. One solution to this deficiency is to extend the network protocol to include an environment field. Each point would then have an associated field defining the environment where the point is located. This blends itself extremely well with the RTAP system because an RTAP application can transparently be connected to several RTAP 
environments. In addition to extending the network protocol, the initial "connect" message should include a list of all environments that are represented by points within a SOM screen. Therefore, when a SOM Server receives the extended "connect" message, it can establish a connection to each of the environments.

Associated with each RTAP point is a data quality attribute. Further discussions need to transpire on how we should utilize this attribute. Furthermore, the current concept of port status needs to be addressed.

\subsubsection{Modifications to SOM}

This section describes the modifications that were made to SOM in supporting the RTAP integration effort. Modifications were made within two distinct areas; the network API and the model files. No modifications were necessary to the core component of the SOM application.

Data Alignment: The network API that was used for the evaluation implementation was derived from an earlier investigation performed by a Military Research Associate. This earlier investigation focused on changing SOM from a polling implementation to an event driven implementation. Because the network protocol was developed using TCP instead of DECnet, we elected to utilize this work as the foundation in developing the network protocol for the RTAP implementation. However, in order to utilize this pre-existing work, several changes needed to be incorporated. First and foremost, was changing the data within the network protocol to be naturally aligned. For efficiency reasons, some systems expect data types to be naturally aligned on even word boundaries. The VAX architecture allocates exactly what is required without regards to alignment. Misalignment of data on systems expecting data types to be naturally aligned can result in an underflow, overflow, or divide by zero exception to occur. Because the earlier investigation was between similar architectures (i.e., VAXes), natural alignment was not an issue. However, for the RTAP implementation, data on natural boundaries was an issue. Therefore, the network protocol was modified to force the data within the network structure to be naturally aligned.

Data-Word Order: System vendors store the order of a data-word in different formats. Data-word order refers to the internal storage representation of integer and floating point numbers. No standard exists for the order of data-word; therefore, non-uniform formats exist among different systems. This issue was addressed in the evaluation implementation by developing a set of conversion routines that can be used to change between differing formats. Before data was transmitted across the network, a conversion process was performed to resolve data-word order. Refer to Appendix $C$ for further details.

Floating Point Representations: Most computer architectures have standardized on the IEEE-754 floating point specification; the VAX architecture does not. The 
VAX architecture employs its own representation. Therefore, in conjunction with the data-word order issue, a set of routines were developed to convert between the two representations. Before data was transmitted across the network, a conversion process was performed to resolve floating point representation. Refer to Appendix $C$ for further details.

Model Files: The model files needed to conform to the new ASCII point names that were derived for the Heater Control and the Dye Combination Wall RTAP databases. Furthermore, we standardized upon uppercase characters for representing the ASCII point names within the model files and the RTAP environments.

Miscellaneous: The following represents miscellaneous issues that were resolved within the evaluation implementation.

- Standardized on lowercase character for representing an RTAP environment name.

- Once the SOM Server re-establishes communications back to SOM, the connection to the SOM Server Launcher's "well known" address was closed.

\subsubsection{Future Considerations}

The following issues should be considered in any follow-on work pertaining to the integration of SOM into RTAP.

- The evaluation implementation continues to send updated values to SOM even if the SOM screen is iconified. To decrease the performance penalties associated with this issue, SOM should not receive updated values when the screen is iconified. Once the screen is expanded from an iconification, SOM should receive all points that it is monitoring to reflect any values that were updated during the iconification.

- Perform a major re-write of the protocol and associated software used to communicate between SOM and RTAP. As previously stated, this protocol was developed to support an early attempt of changing SOM from a polling implementation to an event driven implementation. The primary reason for the re-write is that the protocol and support software is not up to production quality.

Furthermore, several deficiencies exist that need to be resolved. These include:

- Reducing the number of bytes that are sent within a network message. Currently, each message contains 161 bytes. However, the majority of this space is never used. Though this issue did not impact the evaluation implementation, it would impact network traffic within a production implementation. 
- The current software to support the protocol will "hang" SOM in the event SOM transmits a point that is not defined within the RTAP database. The reasoning is that when a "declare interest" message is sent to the SOM Server, the SOM API waits to receive the initial value for the specified point before control is returned to SOM. In the event SOM transmits a non-existent point, the SOM Server will ignore the point and never send an initial value back to the SOM API. In this case, control will never be returned to SOM and the program will appear to be "hung". This is because the SOM API is awaiting for an initial value from a non-existent point. Therefore, appropriate logic needs to be incorporated into the SOM API to handle this event.

\subsection{A Comparison of RTAP and the System GUI}

The purpose of this section is to compare the schematic display capabilities of RTAP and the System GUI in order to determine whether RTAP can replace the System GUI in a new control system designed to replace the current one. The functionality of each system is described. A comparison of features that are implemented by the System GUI with that which is provided by RTAP is given. The description of each system's capabilities and their relative comparison is the most important thing to consider in making the decision. Other points to note are each system's ability to interface with other applications, each system's ability to be extended beyond its current capabilities, and the ease with which each system is learned, understood and tested.

\subsubsection{RTAP}

RTAP enables users to interactively create schematics and control panels using the applications called RtapScBuilder and RtapCpBuilder. A third application, called RtapSchemat $X$, loads the schematics and control panels, connects them to the database, animates the displays in response to changes in the database, and executes commands from the user.

\subsubsection{User Interaction with RtapSchematX}

Commands are actions performed on behalf of objects such as buttons or symbols on a schematic. Objects with associated actions are called hotspots. These actions are performed by the RtapSchematX program whenever the user moves the mouse cursor over a hotspot and clicks MB1. The mouse event that triggers a hotspot is by default MBI single click, but can be changed to MB1 double click, MB2 single click, MB2 double click. The shape of the mouse cursor can be changed for any hotspot.

These commands enable the user to pop up a schematic in a new window, or replace a schematic in the current window, and refresh the data on a schematic. 
Users can also acknowledge alarms, use a control panel to change data in the database, start the trend application called RtapPlotDisp, start and send a text message to other applications, popup a menu, and exit RtapSchematX. $R$ tapSchemat $X$ can receive actions via the RTAP message queue. This makes it possible for another application to cause RtapSchematX to perform an action, such as display a schematic or control panel.

\subsubsection{Dynamics}

RTAP supports a small set of behaviors for the objects on its schematics. These behaviors, or dynamics, are performed in response to changes in the value of attributes in the database. RTAP supports three types of dynamics : analog, format, and fill. The analog dynamic is used to associate a change in value of an attribute in the database as a change in color, line style, interior style, and text style of an object. The format dynamic is used to display a value as a text string. The fill dynamic is used to display a value as a percentage fill of a polygon. Lots of other dynamics are supported in the RTAP Application Programming Interface (API), which would allow for the development of applications to replace RtapScBuilder and RtapSchematX. It would not make sense to replace either application singly because the RtapScBuilder defines the layout and behavior of a schematic, and the RtapSchemat $X$ uses the schematic to display and animate it.

\subsubsection{System GUI}

The System GUI enables the user to interactively create schematics using the Draw application within the commercial product called SL-GMS. SL-GMS, the SL Graphical Modeling System, is a graphics software package that is used to create and animate graphical screens. SOM is the custom application developed by CNC which uses the SL-GMS function library to load the schematics, connect them to a database, animate the display in response to changes in the database, and respond to commands from the user. The animation that SOM can perform greatly surpasses that provided by RTAP, because more functionality is provided to do this by SL-GMS. SOM's method of interaction with the user allows the user to point and click at objects on schematics, and perform a command on them.

\subsubsection{User Interaction with SOM}

SOM uses a different scheme to interact with the user than that used by RTAP. Users access commands by selecting menu items from a main menu bar located at the top of a SOM window. These menu items are as follows : System, Control, Trend, Print and Help. Selecting a menu item results in the display of a pulldown menu. Commands accessible from the Control and Trend pulldown menu are dependent upon the user selecting one or more objects on the schematics, whereas those from the System, Print and Help pulldown menus are not. 


\subsection{Selecting Objects to Control and Trend}

Before selecting a menu item from either the Control or Trend pulldown menus, the user must select one or more objects. This is done by pointing to an object with the mouse pointer and clicking MB1 to select one object, or depressing the shift key at the same time to select multiple objects. All instances of an object are selected by default, which provides for consistency across schematics. When an object is selected, it is highlighted with either a dashed box or hatch pattern. From the Control and Trend pulldown menus, the user can launch built-in control panels, the ODC Control Panel, the Video Panel, the Thermal Control Panel, or the Trender. Each of these applications is sent information about the objects selected by the user, such as the names of data values in the database, camera names, and ODC device labels. The user can also double click MB1, which is interpreted to mean select the object(s) and perform the default action, which is to launch a control panel. Lastly, the user can turn detailed labeling information on or off on all schematics from the Control pulldown menu.

If no object is selected, the user is prevented from performing any object-based actions. This is accomplished by modifying the sensitivity attribute on the corresponding menu item. If a single object is selected, the user can trend it. If there is a control panel or the Video Panel associated with the object, the user can display it. If the user selects more than one object, the user cannot display a control panel. If no more than four objects are selected, the user can trend their data. If no more than thirty objects are selected, and any of those objects are cameras or sensor packages, the user can display the Video Panel. SOM maintains communication with the ODC Control Panel, Video Panel and Thermal Control Panel, enabling these applications to receive future selections from the user.

\subsection{Displaying and Printing Schematics}

The System pulldown menu lists the name of each schematic that can be displayed. There is an optional submenu that can be activated from each System menu item, which allows the user to select a schematic that represents a more detailed view. Schematics can be changed at any time from the System pulldown menu. A hierarchy of schematics is not imposed on the user, so any schematic can be accessed while viewing any other schematic. Users can also refresh the current schematic from this menu. An alternative method of changing schematics is also supported. The user can double click MB1 on a schematic button, located anywhere on a schematic, which will replace the current schematic with the one associated with the button. The Print pulldown menu allows the user to either save the current schematic in a file or to send it directly to a printer. Schematics are saved and printed in color postscript format. The Help menu item displays information about SOM. 


\subsubsection{Dynamics}

SL-GMS supports a large set of behaviors for the objects on its schematics. These behaviors, or dynamics, are specified as actions applied to objects whenever the object's associated data changes value. The resulting graphical changes include attribute changes (26), transformations (11), graph changes (11), and special actions (7). A summary of these changes is included in the following list : position and movement with $X, Y$, and $Z$ controlled independently; scale and size of objects; rotation of objects from any designated reference; full and partial fill of circles, closed splines, rectangles, and polygons; color; visibility; line style and width; radius of a circle; text, including numeric and string formats; text font and size; graph axes and traces, linear, log and time (calendar/clock); charts, bars, and pies; calls to application-defined functions.

The dynamics are specified using actions, in a script language that resembles $\mathrm{C}$. Actions can be unconditional, conditional, or implicit. An unconditional action is one that is applied whenever the dynamic behavior of an object is evaluated. A conditional action is one that is applied whenever the data associated with the object is equal to a constant or falls within a certain range. An implicit action is one that restores the object to its original state if no actions are applied. Actions depend upon data values, which can be variables, integer, real and string constants, unary and binary expressions $(+,-, *, \mid, *)$, relational expressions $(>$, $<,>=,<=,==$, \&\&, | I, !=), math functions available in the $C$ library, and userdefined functions.

\subsubsection{Comparison}

Both systems are functionally divided in the same way, meaning that there is an application to draw screens and one to display screens. This division of functionality allows the work of implementing the user interface to be done interactively, by drawing schematics and specifying how they should be animated, without writing custom application code. The features that are implemented in each system's applications are quite different. RTAP has a third component which is not available in the System GUI, which is the application that interactively creates simple control panels. These are control panels that read from or write to the RTAP database. SOM has a number of simple control panels built-in, and has the ability to start and communicate with applications that implement complex control panels, such as the ODC Control Panel, Video Panel, and Thermal Control Panel.

A key distinction between the two systems is that SL-GMS supports a wealth of dynamic behaviors that have not been fully utilized in schematics that are being used in the control system today, whereas those provided by RtapScBuilder cannot supported those same schematics. The method of interacting with the user that is supported by RtapSchemat $X$ is very limited in comparison to that 
supported by SOM, and cannot be extended. Since SOM was developed by CNC, it can be changed to include any additional features that are needed. It is worthwhile to point out that the design of SOM's interaction with a database allowed us to make minimal changes to SOM in order to get data from RTAP instead of the current control system database.

\subsubsection{Features Absent in RTAP}

Overall, RTAP lacks many of the key features provided in SOM and SL-GMS. Most importantly, it does not provide a method of selecting objects on schematics, then performing an operation on them. This makes it impossible for users to pick data values on a schematic to trend, or to pick cameras and video signals from sensor packages to display on their video monitors, or to pick mirrors to move in order to align a laser beam. It is also important to note that the dynamics provided in RTAP are inadequate to implement many of the behaviors designed into existing schematics, such as dynamic pie charts on the SRG/RGA schematics, highlighted mirror and beam paths, and the ALFI shutters (multiple layered, grouped objects that change color independently), to name a few. RTAP dynamics operate only on database values, whereas SL-GMS dynamics use constants and user-defined variables, making it possible to describe complex behaviors. It is evident that RTAP and the System GUI were designed to meet different goals.

\subsection{Display Capabilities}

The following is a list of display capabilities that are supported and utilized in SOM and its schematics on the production systems, but that are not supported by RTAP.

- Embedding graphs on schematics.

- Using constants, user-defined variables, unary and binary expressions, relational expressions, math functions and user-defined functions in dynamics.

- Grouping objects.

- Translating and rotating objects dynamically*.

- Setting the visibility and detectability of objects dynamically.

- Modifying line width dynamically.

- Using fill patterns.

- Displaying date and time on schematics.

* RTAP can simulate translation and rotation to some extent. It requires that the user draw the object in each position to which it will be rotated or translated, then use color changes to make the current position visible, and all others invisible. SL-GMS has a simpler method that results in a much better looking animation effect. For example, if you want to move a needle in a volt meter, 
specify the range in volts $(0$ to 120$)$ and the arc that the needle with sweep ( 0 to -90 degrees). SL-GMS will automatically interpolate the rotation :

volts

$=0: 120$

rotate $0:-90$

\subsection{Interaction Capabilities}

The following is a list of interaction capabilities that are supported and utilized in SOM on the production systems, but that are not supported by RTAP.

- Selecting and highlighting objects, and using information about those objects as input to commands.

- Starting application process, such as the ODC Control Panel, Video Panel and Trender, and sending them information about objects selected by the user.

- Maintaining two-way communication between SOM and an application that is tailored to meet the requirements of the application.

- Using a main menu bar, pulldown menus and submenus to perform commands.

- Saving and printing schematics.

\subsubsection{Features Absent in the System GUI}

There are several important features provided in RTAP that are not supported in the System GUI. These are : support for interactively creating control panels; a method of interacting with the alarm system from a schematic or control panel; integrating building schematics with the database. RTAP has an application called RtapCpBuilder, which enables users to interactively create simple control panels. It is a straightforward job to create a new control panel and have RtapSchematX display it. The same is not true for SOM. If the new control panel is different from the built-in ones, code must be written for SOM to create and manipulate the control panel. Secondly, RtapSchematX has the capability to enable users to acknowledge alarms from a schematic, which SOM does not do. RtapScBuilder allows the user to link symbols on a schematic to points in the database in a straightforward manner. The user is prompted for the DB Path from a dialog box displayed when User Data is selected from the Tools menu. The way in which data is linked to objects on SOM's schematics is through a user-defined text field in the schematic called UserData. This field can contain any sort of information. SOM uses it to tie objects to a database. There isn't any help or verification of this string because the format is open. 
Several other features that are worth noting are the way that RtapSchematX changes the mouse pointer when it moves over a hotspot, the fact that it can can start any application and send it a text string, and that it can receive a command from another process and perform the function. These are things that SOM does not do.

\subsection{Display Capabilities}

- Creating and animating new control panels without writing code.

- Changing the mouse pointer when it moves over a hotspot.

\subsection{Interaction Capabilities}

- Acknowledging alarms.

- Starting any application and sending it a text string.

- Receiving a command from any application and performing the function.

\subsubsection{Conclusion}

It is clear that RTAP could not replace the System GUI in a new control system designed to replace the current one. It lacks too many of the display and interaction features with which users have become familiar and rely upon to perform their work. The System GUI is extensible enough such that it can support new features that are deemed necessary to add. The areas in which the System GUI is weakest in are interactive creation of simple control panels, integration with an alarming system, and training. This is mainly due to the fact that the System GUI was not developed as a commercial product. Attention needs to be directed to these areas in order for people outside of this program to use the System GUI.

\subsection{Trending}

The RTAP plot display program, RtapPlotDisplay, provides for viewing historical data. A utility program, RtapTPConfig, is used to interactively configure trend plots. Plots can be displayed on any Xwindow device or graphics terminal, and printed on any Printer Control Language (PCL) printer (such as an HP LaserJet or PaintJet).

Three alternatives are considered when designing trend plot configurations. In the first method all trend plots have the same general appearance, consisting of the same number of signals and scaling, but displaying different data points. In this method the plot points are grouped under the plot configuration point in RTAP's database. The second approach is to configure several different trend plot types, grouping them under the plot configuratiion point. The third method 
is to distribute trend points throughout the RTAP database, each referencing a specified plot configuration point.

\subsubsection{Advantages}

The main feature of using RtapPlotDisplay for trending is that some historical data is stored in the database (in a history table) and can be displayed at system startup. By organizing the plot hierarchy according to the types of plots that are needed, plot points can be located in the database near their configuration points or distributed throughout the database. It is possible to dynamically (while the environment is running) add history and plot configuration points to the database.

\subsubsection{Disadvantages}

RtapTPConfig does not allow manipulation of all plot configuration options. To alter the appearance of existing trends, a new trend configuration point is created and the new trend is invoked.

An RTAP display license is required for each $X$ server used to display trends initiated with RtapPlotDisplay (it is acutally RtapXColorMngr that uses the license).

A plot historian configuration point must exist in the RTAP database. This point contains database address cross references for signals to be trended and an array to store historical data for each address of interest. One disadvantage is the plot configuration files. Each trend plot configuration requires a plot configuration point to specify the data to be trended. Trend plots are currently limited to a maximum of eight signals.

One method of reducing the amount of historical data stored in RTAP's database is to allocate space for some maximum number of unique points to be trended in an RTAP history point. This method only provides for a localized history point that contains some number of database point addresses. The disadvantage is that history points would have to be manipulated (changing the cross references) to choose the points of interest.. If using RTAP's plot display utility, some predefined set of trend configurations would have to exist.

\subsubsection{Future Considerations}

An alternative method would be to not use RTAP's trender, but to use some convenient and flexible method of getting the RTAP data into a plotting utility. One method we looked at is to use $\mathrm{Scl}$ and $\mathrm{Scl}$, products from Computerized Processes Unlimited (CPU). Scl and SclX are based on Tcl/Tk (a tool command programming language and windowing shell developed by Dr. Osternhout at the University of California, Berkeley). Scl provides extensions to the RTAP database in the form of a high level programming (Unix Shell) language. I was 
able to produce nice looking trend plots with SclX. However, an RTAP database history point is still required to specify which signals are available for trending. One advantage of this method is that no RTAP display license is required for each Xserver. Sources for the script approach to trending are on sdxs02 in the /users/rtap home directory and have been included in the appendix of this document. CPU's products, including their "Trend" script are located in /usr/var/cpu/bin .

An example trend plot produced with CPU's "Trend" Scl script.

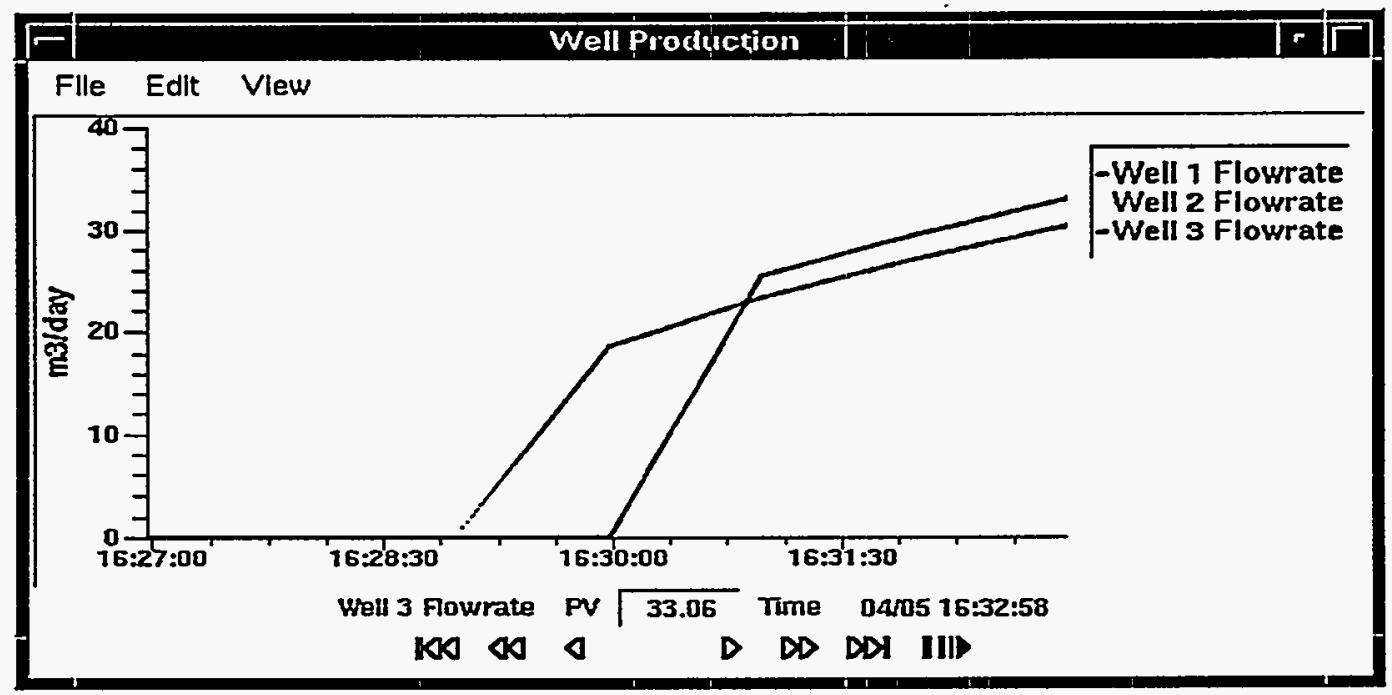

The little buttons at the bottom function like a VCR's buttons, allowing fast forward, rewind, and pause. The plot annotation and ticks are totally under user control through the invoking script file.

\subsection{Future Considerations}

An investigation should be performed in identifying the benefits and regrets of using $\mathrm{X}$-terminals over workstations. 


\subsection{Communicating with Foreign Devices}

\subsection{Scan Tasks}

Most of the information in this section comes out of two RTAP manuals: Chapter 4 "Configuring a Scan System" in the RTAP Integration Manual and Chapter 6 "Adding a Scan Task" in the RTAP Programming Manual. The scan system consists of a single scan manager, one or more scan tasks that it oversees, and a set of routines that are available for developing user-written APIs. The scan manager coordinates the scan tasks and manages the Scan Shared Memory Area (SSMA). Scan tasks handle all communication between scan devices and RTAP through the hardware communication ports. Each scan task communicates with a specific type of scan device or FEP (Front End Processor). Each scan device has I/O points wired to instrumentation and control devices. The scan system gathers input data from these devices and stores it in the SSMA. Scan links in the database processed by the Calculation Engine tell RtapMQDBM Database Manager how to map the data from SSMA into the database. Scan tasks can also send data out to an FEP.

Chapter 6 of the Programming manual points out that in some instances it can be more advantageous to write a server application as opposed to a scan task. The book suggests that if the field device is at a very high level of communication (i.e. LAN based) or if optimization and speed are very important, you might want to use a dedicated server program. It suggests one might want to use the scan task if: 1) the field device is fairly dumb; 2) the field device requires low level communication control such as retries and timeouts; 3 ) the field device fits well into the hierarchy of the scan config branch of the database ;4) the advantages of decoupling and SSMA are important; 5) deadbanding is required; 6) a generic $\mathrm{CE}$ (Calculation Engine) interface with the scan links is desired; 7) a generic programmatic interface to all field devices is desired.

For a purchased scan task almost all of the additional effort is put into configuring the scan task. A scan system is a hierarchical system and is represented in the RTAP database as such. There is only one scan system running in an RTAP environment although it's possible to have multiple environments on a single computer. The scan system is run by the Scan Manager which is represented in the database as scan config (hanging off of root). The next level is the communication port and there can be a maximum of $32 \mathrm{comm}-$ ports. Each port is configured to handle a particular kind of communication protocol, baud rate, etc. on a single communication medium such as RS-232, LAN, etc. Each comm-port can have up to 255 similar end-devices associated with it. The next level is the scan device and it can have up to 65,535 input and 65,535 output points. These points are represented in the Scan Input and Scan Output Tables and is the lowest level of the hierarchy. 
What tasks does the Scan Manager do? When the Scan Manager is first started, it validates the scan system configuration information in the RTAP database and uses this to allocate the SSMA required for input scanning. It then schedules a scan task for each comm-port using the process scheduler. Next the Scan Manager requests the Timekeeper to send periodic alarm-clock messages to the appropriate scan tasks for all poll/PRBX (polled report-by-exception) types that are configured to be awakened. Poll and PRBX periods should be set appropriately for the speed at which the scan task can retrieve the required data from the scan devices. Since the scan task may have to communicate over a relatively slow asynchronous line (possibly involving retries) and then trigger RtapMQDBM to update the database, the scan task may not be able to keep up with its messages. When multiple poll requests are received at the same time, they are merged into a single poll. If the input message queue gets too full, the scan task attempts to clean it up by ignoring poll and PRBX requests from the Timekeeper. If this still does not solve the problem, it starts rejecting requests from APIs. The Scan Manager also cleans up scan system resources when the environment terminates.

Each level of the scan system hierarchy is configured in the database. The Scan Manager level is represented by the scan config database point. In this point is a table of the scan tasks that you want to run in your system. The next two levels, the comm-port level and the scan device level, have device independent points that are required and device dependent points, some required and some optional. Some device dependent values are the same fields at both the commport level and the scan device level. The scan task philosophy is to move a field value to the highest level if it is common amongst most of the scan devices. The scan device field value can override the comm-port fieid value if it needs to be different. Some of the fields to configure in the comm-port level are: device type which is the index into the scan config point's scan task table defining what scan task to use; retry count which is the number of times you want the scan task to retry communication with an enabled scan device before reporting failure; and reply timeout, the number of milleseconds that the scan task waits for a reply from a scan device before retrying the communication. These are just a few of the fields to give a sample of what's there.

The next level is the scan device level. Some of the fields to configure for it are: device poll types which is a mask that enables/disables the different polling times that are set up in the scan input table (which is at the next level); scan input control which enables or disables polling; and failure threshold which is the number of times communication with a scan input point is attempted before the SSMA quality-status flag is set to failed. Again there are many more fields at this level.

The scan device points are defined in the Scan Input and Scan Output Tables. Some of the Scan Input Table fields are: point control which enables or disables scanning for that point; RTAP DE type which is the data type for this element in 
the SSMA; and deadband type which is one of 3 values (absolute or value deadbanding, percentage deadbanding, or no deadbanding). Again these are just a few fields that need to be configured. When a database point is created to accept the data being read from a scan device, a scan link is defined in the attribute definition field for the point. In this scan link is the index (row number) into the Scan Input Table that contains information about this point, such as where the data is located in the SSMA.

There is a lot of configuration to be done for the scan config branch of the database. It seems that a system can be finely tuned using all of the different configuration choices at all levels. It will take some experience working with RTAP and scan devices to know what things should be tuned.

\subsubsection{Heater Control Scan Task}

At this time RTAP sells 3 Allen-Bradley (A-B) scan tasks: the Data Highway Scan Task which interfaces the RTAP host to a PLC via an RS-232 link using the Data Highway Plus protocol, RtapAbPiScan which allows communication between the RTAP host and a Pyramid Integrator (PI) over Ethernet using the TCP/IP protocol, and RtapAbKtScan which provides communication to a PLC that is on a Data Highway Plus network via an A-B KT/B card that is installed in an $\mathrm{HP}$ 9000 series 700 computer. Both RtapAbPiScan and RtapAbKtScan require that a version of A-B DTL (Data Table Library) be running on the RTAP host computer. DTL is a library of routines that are used to exchange information (data, commands, etc.) between an RTAP host computer and an A-B PLC.

Reported first will be the two scan tasks that were not used for the RTAP evaluation--the two DTL scan tasks and a little about hardware configuration. The RtapAbKtScan requires an EISA slot for each KT card installed on an HP. A maximum of $4 \mathrm{KT}$ cards can be installed in a single $\mathrm{HP} 9000$ series 700 computer and RtapAbKtScan can access up to $4 \mathrm{KT}$ devices. There is no limit however on how many logical scan devices that can be addressed via the KT card. DTL supports multiple scan tasks but limits each task to 40 asynchronous read/write requests in progress at any one time. This is true for both DTL scan tasks. For this scan task, DTL is restricted to transferring 240 bytes from a Data Highway device (the PLCs). This determines how large the element count of a vector can be as no attempt is made to break large requests into small requests. One of the disadvantages that was observed with this scan task was that one KT card for every 4 devices is required, and this seems cumbersome and possibly expensive. One may however be able to reach more than the 4 PLCs if others are hooked together on the same Data Highway Plus network. This is unclear. When using Data Highway Plus as the communication protocol, slower response should be expected than when using the DTL Ethernet interface. Data Highway Plus runs at a baud rate of $57.6 \mathrm{~K}$. 
The RtapAbPiScan task can run on the HP 9000 series 300, 400, 700 and 800 computers. It supports up to 40 A-B PI units on a given network. Each PI unit must include a Resource Manager (RM), Ethernet Interface (EI), and a power supply. The EI module requirement is why we can not use this scan task with our current PIs; we have no free slots left in which to plug in an EI card. DTL manages all communication with the PIs on the network, and the PLCs connected to the PI via Data Highway Plus. Each EI card is capable of serving up to 10 simultaneous DTL clients so there could be 10 RtapAbPiScan tasks running. The limit of 40 asynchronous read/write requests in progress per scan task applies here too. DTL is restricted to transferring 2048 bytes from a PI. Again this puts a limit on the element count of vectors being shipped. Speed and larger vectors seem to be big advantages for the PI scan task. However, at this time the PI scan task has not been implemented to its full capacity. We understand that the PI scan task can service 5 requests per second. CPU is currently working with Allen-Bradley to improve this performance (by enhancing the scan task) and something may be ready in a few months. We have proposed replacing the PIs with some newer A-B PLCs that have an Ethernet port on the front of them. It is not known (at this time) if there is an RTAP scan task for theses new processors or not. The addressing is different on the PIs than it is on the other PLCs so the RtapAbPiScan task probably would not work with the new processors.

The scan task we used to communicate between RTAP and a PLC 5/15 for our evaluation was the Data Highway Scan Task. An Allen-Bradley 1785-KE Data Highway Plus Communications Interface Module was installed in a PLC 5/15 and an RS-232 link was hooked up between this module and the HP machine called Snailbait. A subset of the Closed Loop Heater Control system (about 50 points) was implemented in the RTAP database and in the scan input and output tables of the Data Highway Scan Task. We were successfully able to control and monitor points from the PLC using the RtapPtDisplay tool, using SOM, and using the RTAP schematics we created.

The first step was to get the A-B 1785-KE installed in the PLC 5/15 and hook an RS-232 cable up between the PLC and Snailbait. After that it was time to configure the Data Highway Scan Task. First, the different levels of configuration described in section 6.1 Scan Tasks were accomplished. This consisted of scan config point, comm-port level which was DH_port, and scan device level which was PLC5. Next, all of the monitored points in the Scan Input Table and the control points in the Scan Output Table were configured. All of the devices were polled at once a second, with no noticeable performance problems. However, no true performance tests were done on the system (simulating changing values in the PLC, changing the deadband value, etc.) Currently, once a second is the fastest poll rate, but at the latest RTAP User's Group (RUG) meeting it was announced that this had been improved to 2 Hertz. To send control without an API, one enters two values in the database: the value to be changed and the trigger value. When the trigger value is set to a one, the 
control value is sent to the PLC. There is a scan output definition on the trigger attribute that is the index into the Scan Output Table for the control point. Doing control with an API needs to be investigated. This was explored some using SCL (CPU's Sequence Control Language). An event handler was attached to our control point. When the event message was received (the write occured) the value was sent to the PLC.

From what was seen in the evaluation system, what has been read, and what we have been told from our consultant, it is improbable that the Data Highway Scan Task will be able to handle the load in the Production System. We need to get an Ethernet Scan Task and see if it can perform under heavily loaded conditions satisfactorily. There were no problems with tasks bombing or the environment going away during this evaluation. This was reassuring as we had that experience during the RTAP training class. Once the Scan Manager was activated, the scan system hummed away without interruption.

\subsection{Server Tasks}

As previously described for the SOM Server Launcher, communication with the Optical Device Controller (ODC) is facilitated through a server routine that establishes a socket connection to the ODC and uses RTAP's message queue to make the RTAP database to ODC connection. Software for these processes has been completed and tested.

\subsubsection{ODC Server Launcher}

In exactly the same design as used for the SOM Server Launcher, the ODC Server Launcher is a routine, running in the RTAP environment, that listens for a service connection request from an ODC through a dedicated TCP/IP socket. Upon receipt of an ODC connection request, an ODC Server process is started. This process then listens to a designated socket and connects to the RTAP message queue to pass messages between RTAP and the ODC.

\subsubsection{ODC Server}

TheODC Server receives network messages from the ODC and decodes each message for the specific action required (based on a message type field). The resulting action is then used to compose the appropriate message for RTAP's message queue. The message types and attributes were worked out in the software design phase of the ODC Server. See the source code for details.

\subsubsection{Future Considerations}

Scaleability is one issue to be considered when building messaging systems. One advantage of having separate server processes for each ODC to RTAP connection 
is that the workload is distributed among the machines where the server processes are invoked. The down side is that network messaging is not coordinated among these server processes. The number of network messages sent to the RTAP message queue at any given time is not predictable. Using a single process for all communication connections makes the server software considerably more complicated and localizes connection processing to a single node. An alternative might be to implement communication as a polling operation where possible. Using a specified poll rate that is acceptable for window and database updates, then a single network message could consist of all associated attributes of an ODC or SOM. The server process would have to parse the message and update those attributes that have changed, ignoring information that is unchanged since the last poll. The upshot is that the number of network messages at any given time can be predicted, with the minor inconvenience of having unused data sent over the network. 


\subsubsection{Modifications to Existing Software}

The RTAP implementation of the ODC FEP Server uses a multitasking ADA application as shown in figure 6-1.

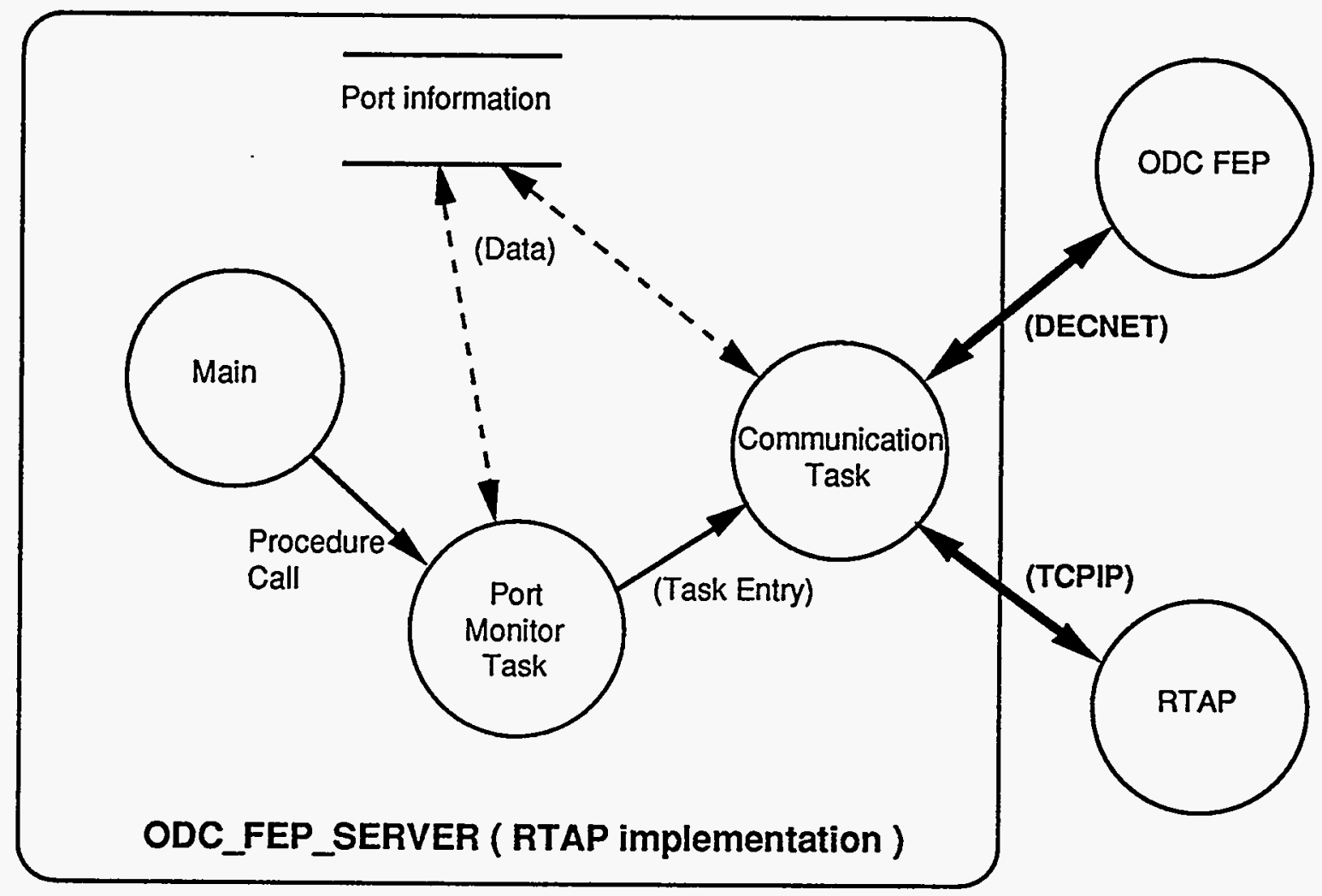

Figure 6-1 : ODC FEP Server ( RTAP Implementation)

This is basically the same design used with the State tree implementation with some slight modifications.

The ODC FEP Server program has three ada modules that implement the specific functionality to communicate with ODC FEP's. These modules are

ODC_FEP_SERVER \{ main program body \}

ODC_STRÜCTURES \{ spec and body \}

FS_COMM \{ body only\}

\subsubsection{ODC_FEP_SERVER Module}

This module was completely ripped apart but fortuanately it was very small to begin with. This module initializes the ODC IO Library, then loops through the known ODC FEP's and makes a call to the FS_COMM module to start a communication task for that FEP. After completetion the program goes into an infinite delay loop. 


\subsubsection{ODC_STRUCTURES Module}

This module contains data structure definitions and initialization code that are used and called from procedures in the FS_COMM module. Modifications to this module were the addition of a data structure used to store RTAP specific information on the ODC points to be archived and a new initialization procedure used to fill this data structure. All code related to state tree communications was left in. This module is compatable with both RTAP and State tree implementations of the ODC FEP Server.

\subsubsection{FS_COMM Module}

Most of the code in this module was reused but modified to meet the requirements of the RTAP implementation. All the data server related code was ripped out. Referring to figure 6-1, the FS_COMM module implements the port monitor task and the communication task. The port monitor task is used to initiate communications with an ODC and then start the communication task that is to perform the actual transfer of data between RTAP and ODC FEP's. This action is taken in response to a call from main to start ODC communications. Once communications have been established the port monitor task monitors the state of the ports restarting those that terminate. The Communication task is used to implement the communication protocols between ODC's and RTAP fep servers and for the transfer of ODC data to the RTAP database. There will be one communication task for each ODC RTAP communication link. 


\subsection{Alarming}

Alarming system is an important component in the Supervisory and Control System. It provides timely information and warning to operators which help prevent interruption of plant operation due to equipment failure and personnel safety due to unsafe conditions. The alarming system must be reliable, efficient, and easy to use. Otherwise, It will not be used at all. To understand the requirement and the architecture of the U-AVLIS alarming system, documents of the system were reviewed and informal discussions held with several knowledgeable people. The requirements form the basis for evaluating RTAP alarm system capabilities that we had only class room training experience.

The U-AVLIS alarming system is a complex system with multiple layers and segments. Alarms are classified into levels of severity that require different acknowledgments and responses from the responsible operator. Alarm history files are archived while selective report may be generated according to a set of sort criteria. The system was designed and implemented with software engineering procedures. However, several areas of improvements are noted.

- Better integration of alarm notifications and handling between different levels and segments of the control system.

- Improve the responsiveness and reliability of alarm notification.

- Improve the MMI and GUI so that operator can conveniently access detail alarm information from a single console.

- The current alarm system is very CPU intensive. Efficiency must be improved.

RTAP Alarm capability is one of the strengths of the new release. Alarm system was completely re-designed which boosted the alarm detection and processing by 6 to 10 fold. The determination of alarm conditions is within the alarm database that eliminated the communication delay between processes. Other built in features of the RTAP alarm system include audio output, ability to execute another UNIX command, and starting up other processes. Overall, RTAP provides a reasonable set of alarm processing modules and has additional hooks for user customization.

Alarm system requirements for a production facility will most likely be more stringent and operational safety rules will be different from a prototype facility. Therefore, interviews with appropriate personnel in each major sub-system to gather pertinent information must be conducted. A requirement document reflecting the specification of needs will be prepared and reviewed by users for accuracy. The next step is to design the architecture of the alarm system with special attention to the four areas mentioned above. The implementation of the system should take advantage of the RTAP standard capability and special applications will be developed for customization. Test cases need to be 
developed to verify the functionality and performance of the system. Software engineering practices including up-to-date documentation should be followed.

In addition to interviewing responsible personnel in all major sub-systems, a more in depth understanding and familiarization of the RTAP environment; in particular, the alarm management system is needed. This will help in the design of the architecture and allow us to fully utilize the capabilities of RTAP. 


\subsection{Performance}

The following performance results were obtained from HP, Calgary, courtesy of Steve Heckbert. These performance numbers are in operations/second, with the following caveats applied:

- not scientific, some systems were busier than others, more or less memory, etc. Lots of differences.

- values are usually the best of 3 or more trials, not average

- IBM is still A.06.20, without RTAP Version 6.3 symbolic database access improvements.

The others are fairly recent 6.3 or 6.4 systems.

- machines used: > DEC 3000 series 500 (alphabet)

HP 715/50 = Pat's (ookpik)

IBM POWERstation 520 (mastodon)

SUN SPARCstation IPX (inuvik)

\begin{tabular}{|c|c|c|c|c|}
\hline & $\overline{\mathrm{DEC}}$ & $\overline{\mathrm{HP}} 715$ & $\overline{\mathrm{IBM}}$ & SUN \\
\hline Local Msg & 3100 & 2400 & 1300 & 650 \\
\hline $\begin{array}{l}\text { RAM read } \\
\text { direct }\end{array}$ & 17500 & 17400 & 10540 & 9000 \\
\hline $\begin{array}{l}\text { RAM read } \\
\text { alias }\end{array}$ & 3000 & 2240 & 780 & 760 \\
\hline $\begin{array}{l}\text { RAM read } \\
\text { path }\end{array}$ & 2100 & 1450 & 415 & 550 \\
\hline $\begin{array}{l}\text { RAM write } \\
\text { direct }\end{array}$ & 1250 & 670 & 400 & 355 \\
\hline $\begin{array}{l}\text { RAM write } \\
\text { alias }\end{array}$ & 920 & 470 & 265 & 235 \\
\hline $\begin{array}{l}\text { RAM write } \\
\text { path }\end{array}$ & 810 & 420 & 204 & 210 \\
\hline
\end{tabular}


HP suggested:

It would be interesting to have these numbers normalized by the specified specmarks for each machine (we'll never be able to compare different platforms one-on-one).

And he found most of the numbers, and I got the rest. These are SPECint92 values, since the parts of RTAP we tested don't use FP calcs:

$\begin{array}{ll}\text { DEC 3000-500 } & 83 \\ \text { HP 715/50 } & 36 \\ \text { SUN IPX } & 22 \\ \text { IBM 520 } & 17\end{array}$

So here are the results again, with each of the numbers being the corresponding one above, divided by the machine's rating.

\begin{tabular}{|c|c|c|c|c|}
\hline & $\overline{\mathrm{DEC}}$ & HP 715 & $\overline{\mathrm{IBM}}$ & $\overline{\text { SUN }}$ \\
\hline Local Msg & 37 & 67 & 76 & 30 \\
\hline $\begin{array}{l}\text { RAM read } \\
\text { direct }\end{array}$ & 211 & $\overline{483}$ & 620 & 409 \\
\hline $\begin{array}{l}\text { RAM read } \\
\text { alias }\end{array}$ & 36 & 62 & 46 & 35 \\
\hline $\begin{array}{l}\text { RAM read } \\
\text { path }\end{array}$ & 25 & 40 & 24 & 16 \\
\hline $\begin{array}{l}\text { RAM write } \\
\text { direct }\end{array}$ & 15 & 19 & 24 & 16 \\
\hline $\begin{array}{l}\text { RAM write } \\
\text { alias }\end{array}$ & 11 & 13 & 16 & 11 \\
\hline $\begin{array}{l}\text { RAM write } \\
\text { path }\end{array}$ & 10 & 12 & 12 & 10 \\
\hline
\end{tabular}

1. Even though the DEC has the best performance in both RTAP and SPECmarks, we get better performance out of the HP than its SPECmarks would predict. i.e. the ratio of RTAP performance to raw machine performance is higher than for the DEC. More of the power goes towards "useful work" (which just means that benchmarks can be misleading, but we all knew that already).

2. The IBM ratio of RTAP to SPECmarks is even higher. It is still running RTAP A.06.20, so its symbolic $\mathrm{db}$ access is lower, but the ratio for the other numbers is the best of all. 
The following are our own performance metrics obtained from testing with the environment "wct" on sdxs02.llnl.gov (an HP 735). All read and write operations were performed on data in memory. For remote environment tests, the environment "wct" on sdxs02 executed the test program to read/write to the remote environment "wctdemo" on snailbait (an HP715). Keep in mind that snailbait is a slower machine than sdxs02. The low numbers associated with remote environment testing are probably due to the relatively slow speed of snailbait. These are good "worst-case" performance numbers, and for the remote testing are not far from HP's performance numbers obtained from an HP 715.

Local environment:

reads/sec.:

Symbolic Address

8330

writes/sec.:

1600

Direct Address

33330

3570

Remote Environment:

reads/sec.:

writes/sec.: 
Appendix A : Technical Issues

- The following environmental variables can be set to produce more detailed error messages during development and/or debugging:

rtERROR_TRACE=all rtSUPPORT_TRACE $=$ on

- Creating an empty database when building a new RTAP environment corrupts the terminal IO upon completion, after environment startup (no keyboard response).

- Numerous environmentals variables related to RTAP should be set in a script file that is sourced when rtap is started, or that is executed prior to starting a desired RTAP environment.

- Displaying RTAP schematics or other RTAP Xwindow processes on non HP platforms may cause problems if the fonts required by RTAP are not installed on the server machine. Similarly, dialog windows may behave differently. For example, using xmodmap to remap the backspace key to be delete is necessary on VAX and DEC workstations that are to display RTAP dialog windows.

- class.config points are prerequisite to using aliases for symbolic database access, a class config point must exist for each point class.

- A troublesome error resulted when the RtapEnvTable started a process and redirected stderr to a log file that was write protected for some users (group permission was used to allow everyone write permission). The log file was in the / tmp directory, and / tmp was emptied as result of a reboot. A user having a different default group then started the RTAP environment, creating the log file with a different group that was previously used. Subsequent users who were denied write permission were unable to start the environment, and the error messages were not too helpful (see below):

The following error messages were spewed out when the above condition occurred. However, these messages were sent to the environment log window, not to the rtap messages window, and as we all know, the environment log window blows away if RTAP fails to invoke the environment. We had to be quick with the mouse to capture messages in the environment log window.

The RtapEnvTable contained an entry something like this:

203 N N N N N 1128 Proc_Foo arg 2>/tmp/log_file 
The file /tmp/log_file did not have write permission for an individual executing rtap. The errors that were displayed, as shown below, were not very helpful in finding the problem:

Error Source: RTAP

Program: RtapDbStartup Env: htrctl

Function: rtGetProcNum

Error: $\quad$ rtE_NOLOC: Process not in local environment

Message: local process 'RtapMQDBM' not found

Error Source: RTAP

Program: RtapMQDBM Procnum: 10 Env: htrctl

Function: NetLS utils

Error: $\quad$ rtE_LICENSE: NetLS licensing error: see RtapMonitor log for details

Message: $\quad$ NetLS utils: NetLS error 0x1d010001

Error Source: RTAP

Program: RtapLicMngr Env: htrctl

Error: $\quad$ rtE_LICENSE: NetLS licensing error: see RtapMonitor log for details

Message: NetLS utils: NetLS error 0x1d010001

htrctl

Function: rtGetProcNum

Error: $\quad$ rtE_NOLOC: Process not in local environment

Message: calling process not part of environment.'htrctl'

Error Detected: Tue May 17 14:24:40 1994

Program: RtapLicMngr Procnum: 0 Env:

Log Message: netls_request_license: License not found in database (network license server/server)

Error Source: RTAP

Program: RtapScheduler Procnum: 1 Env: htrctl

Function: rtMsgRecv

Error: $\quad$ rtE_MSG_SIG: No message received due to interruption by signal

The following message finally indicates that a problem may exist with redirection, and was the clue to the problem. However, this message was not always produced. It was not clear under what circumstances this message was displayed.

Message: signal received while waiting for message

Error Detected: Tue May 17 14:24:42 1994

Error Source: RTAP 
Program: Proc_Foo Procnum: 20 Env: htrctl

Function: exec_runstring

Error: rtE_SYSERR: OS system error

Message: opening file for output redirection

1

I Error Source: OS

I Error: EACCES: Permission denied

Error Source: RTAP

Program: RtapMQDBM Procnum: 10 Env: htrctl

Function: rtCloseSsma

Error: rtE_SS_SSMA_NOT_OPEN: SSMA not open for access

Message: SSMA not open for access

Error Source: RTAP

Program: RtapWDServer Env: htrctl

Function: rtGetProcNum

Error: rtE_NOLOC: Process not in local environment

Message: local process 'RtapTimeKeeper' not found

From the RtapErrowWindow there was no indication of exactly what was wrong.

Starting the RTAP/Plus Tools Palette...

+ RtapRunCmd

PATH=/bin:/usr/bin:/usr/bin/X11:/usr/rtap/bin:/usr/rtap/etc/gettingSta rted/bin:::/users/renbarger/bin:/bin/posix:/bin:/usr/bin:/usr/contrib/bin:/u $\mathrm{sr} / \mathrm{bin} /$

X11:/usr/vue/bin:/usr/contrib/bin/X11:/usr/rtap/bin:/usr/local/X11R5/bin: /usr/local

/bin:/etc:/usr/etc/netls:/usr/var/cpu/bin:/usr/local/rtap/bin:/usr/local/rta $\mathrm{p} / \mathrm{shell}$

:/ usr/rtap/etc/gettingStarted/bin

RTAP_PANELS_DIR=/usr/rtap/etc/gettingStarted/pane

Is RTAP_SYMBOLS_DIR=/usr/rtap/etc/gettingStarted/symbols RtapSchematX $-n-D^{\prime}=+580+$

0 RTAP_Plus_Tools'

Error Source: RTAP

Program: RtapRunCmd Env: htrctl

Function: rtGetProcNum

Error: $\quad$ rtE_NOLOC: Process not in local environment

Message: calling process not part of environment 'htrctl'

+ exit 0

$+[0$-ne 0$]$ 
+ touch / tmp/ActiveEnv.renbarger

Error Source: RTAP

Program: RtapJanitor Env: htrctl

Function: rtGetProcNum

Error: $\quad$ rtE_NOLOC: Process not in local environment

Message: calling process not part of environment 'htrctl'

+ grep - $x$ htrctl / tmp/ActiveEnv.renbarger

$+[0 !=0]$

+ exit 0

+ [ -f / usr/local/rtap/env/htrctl/rtap_log ]

+ echo+ RtapRunCmd -e htrctl

tail -f rtap_log $>/$ dev/pty/ttype

$+[3-$ ne 0$]$

$+[-\mathrm{f} /$ usr/local/rtap/env/htrctl/.RtapEnvLock $]$ 


\section{Appendix B: Development Environment}

Table B1 illustrates the platform specific system software and the associated version numbers used by the evaluation implementation.

\begin{tabular}{|c|c|c|}
\hline Description & Platform & Version \\
\hline HP-UX Operating System & $\mathrm{HP}$ & 9.0 \\
\hline RTAP (Base System) & $\mathrm{HP}$ & 6.31 \\
\hline Allen-Bradley DataHighway Plus Scan Task & $\mathrm{HP}$ & 1.0 \\
\hline C Compiler & $\mathrm{HP}$ & 9.34 \\
\hline VMS Operating System & $\overline{\text { VAX }}$ & $\overline{5.5-2}$ \\
\hline Multinet & $\overline{\mathrm{VAX}}$ & 3.2 \\
\hline Ada & $\overline{\text { VAX }}$ & 3.07 \\
\hline C Compiler & VAX & 3.2 \\
\hline
\end{tabular}

Table B1. System Software

Table B2 illustrates the names and locations of the platform specific files that comprised the evaluation implementation. Files located on the HP system are part of a clustered environment and are available to those systems that have the following devices NFS mounted: sdxs01:/usr and sdxs02:/users. Similarly, files located on the VAX are part of the VAXR cluster and are available to all systems that have the devices mounted clusterwide.

\begin{tabular}{|c|c|c|}
\hline Component & Platform & Directory \\
\hline RTAP Binaries & $\mathrm{HP}$ & /usr/rtap/bin \\
\hline RTAP Environments & $\mathrm{HP}$ & /usr/local/rtap/env \\
\hline RTAP Startup script & $\mathrm{HP}$ & /usr/local/rtap/shell/rtstart \\
\hline RTAP Shutdown script & $\mathrm{HP}$ & /usr/local/rtap/shell/rtstop \\
\hline SCL Startup Script & $\mathrm{HP}$ & /usr/local/rtap/shell/scl \\
\hline CPU's Trend Plot & $\mathrm{HP}$ & /usr/var/cpu/bin/Trend \\
\hline Environmentals Definitions & $\mathrm{HP}$ & /usr/local/rtap/shell/rtenvs \\
\hline Set Environmentals & $\mathrm{HP}$ & /usr/local/rtap/shell/setRtapEnvs \\
\hline SOM Server Launcher (C Code) & $\mathrm{HP}$ & /users/rtap/src/som_server_launcher \\
\hline SOM Server Launcher (Binary) & $\mathrm{HP}$ & /usr/local/rtap/bin \\
\hline SOM Server (C Code) & $\mathrm{HP}$ & /users/rtap/src/som_server \\
\hline SOM Server (Binary) & $\mathrm{HP}$ & /usr/local/rtap/bin \\
\hline Client-Server Utilities (C Code) & $\mathrm{HP}$ & /usr/local/src/utils/client_server \\
\hline Client-Server Utilities (C Code) & $\overline{\mathrm{VAX}}$ & disk\$usera:[elko.ipc_utils.client_server] \\
\hline Client-Server Utilities (Obj Lib) & $\mathrm{HP}$ & /usr/local/src/utils/client_server/libutil.a \\
\hline Client-Server Utilities (Obj File) & $\overline{\mathrm{VAX}}$ & disk\$usera:[elko.ipc_utils.objlib]client_server_utils.obj \\
\hline Data Coercion Utilities (C Code) & $\mathrm{HP}$ & /usr/local/src/utils/client_server \\
\hline Data Coercion Utilities (C Code) & VAX & disk\$usera:[elko.ipc_utils.cvt_data_types.c] \\
\hline Data Coercion Utilities (Obj Lib) & $\mathrm{HP}$ & /usr/local/src/utils/client_server/libutil.a \\
\hline Data Coercion Utilities (Obj File) & VAX & disk\$usera:[elko.ipc_utils.objlib]cvt_data_types.obj \\
\hline Network Msg Coercion Routine & $\mathrm{HP}$ & /usr/local/src/som_server \\
\hline Network Msg Coercion Routine & VAX & disk\$usera:[elko.rtap_eval.som_emulator] \\
\hline SOM (Ada Code and Binary) & VAX & disk\$usera:[public.rtap.som] \\
\hline
\end{tabular}




\begin{tabular}{||l||l||l||}
\hline SOM Network Interface (C Code) & VAX & disk\$usera:[public.rtap.som_if] \\
\hline SOM Model and Config Files & VAX & disk\$Susera:[spencer.inheritance.rtap_eval.thermal] \\
\hline
\end{tabular}

Table B2. Development Software

The above table needs to include the software for:

1) PConfig scripts

2) ODC FEP Server - RTAP compatible

3) ODC Auto populate files

In a collective computing environment there will inevitable exist duplicate files dispersed throughout a development environment. Table B3 describes those files that were shared among the HP and VAX platforms.

\begin{tabular}{|c|c|c|}
\hline File & Directory $(\mathrm{HP})$ & Directory (VAX) \\
\hline client server utils.c & /usr/local/src/utils/client server & diskSusera:[elko.ipc utils.client_server] \\
\hline client server utils.h & /usr/local/src/utils/client_server & diskSusera:Ielko.ipc utils.include] \\
\hline cvt data types.c & /usr/local/src/utils/client_server & diskSusera:(elko.ipc utils.cvt data types.c) \\
\hline cvt data types.h & 7usr/local/src/utils/client server & diskSusera:[elko.ipc utils.include] \\
\hline sda.h & /usr/rtap/src/som_server & diskSusera:[elko.rtap eval.include] \\
\hline sdb.h & /usr/rtap/src/som server & diskSusera:[elko.rtap eval.include] \\
\hline network structs.h & /usr/rtap/src/som server & diskSusera:[elko.rtap eval.include] \\
\hline som api type defs.h & /usr/rtap/src/som server & disksusera:[elko.rtap eval.include] \\
\hline cvt network msg data type.c & Tusers/rtap/src/som server & diskSusera:|elko.rtap eval.som emulator \\
\hline
\end{tabular}

\section{Table B3. Common Files}

In addition, there existed a common data structure that was not defined in one of the common files. Table B4 illustrates this common data structure and the associated source files containing the definition.

\begin{tabular}{|l||c||l||}
\hline Source File & Platform & Structure \\
\hline \hline /users/rtap/src/som_server_launcher/SomServerLauncher.c & HP & som_msg_st \\
\hline disk\$usera:[public.rtap.som_if]msg_svr_api.c & VAX & Connect_msg \\
\hline
\end{tabular}

Table B4. Common Data Structures

Table B5 illustrates two VAX resident header files that contain common data type definitions.

\begin{tabular}{||l||l|}
\hline Header File & Directory \\
\hline \hline client_types.h & disk\$wsera:[elko.rtap_eval.include] \\
\hline network_structs.h & disk\$susera:[elko.rtap_eval.include] \\
\hline
\end{tabular}

Table B5. Common Data Type Definitions 


\section{Appendix C: Utilities}

Two general purpose utilities were developed to support the RTAP evaluation effort. These utilities included routines to establish a typical client-server network connection as well as routines to coerce data between the different data representations. The utilities were designed to be generic; allowing other applications to take advantage of their capabilities.

\section{Client-Server Routines:}

A set of routines were developed to establish a typical client-server connection based on the TCP/IP networking protocol. The first routine is used to establish a network link to a client while the second routine is used for establishing a network link to a server. The routines were designed to be portable between the VMS and Unix-based operating systems. The third-party product Multinet was used to implement the networking capabilities on the VMS operating system.

\section{Data Coercion Routines:}

These routines convert native data types (float(s), int(s) and short(s)) to a format compatible with a particular target machine. The conversions of int(s) and short(s) involve resolving the differences between Endian representations (little vs. big) while the conversion of float(s) involve resolving differences between the VAX and IEEE representations (as well as the Endian representation). The routines operate on unsigned values but work just as well on signed values. The implementation of these routines were designed so that the conversion process is performed once; within the client. The converted values are then transmitted across the network in the correct native format of the target machine. Therefore, conversion is not necessary upon receipt of a network message; only upon sending a message.

The routines support the conversion of the aforementioned data types between the following architectures:

\begin{tabular}{|ll||}
\hline VMS_VAX & $\Rightarrow$ ULTRIX_RISC \\
VMS_VAX & $\Rightarrow$ HPUX_RISC \\
ULTRIX_RISC & $\Rightarrow$ VMS_VAX \\
ULTRIX_RISC & $\Rightarrow$ HPUX_RISC \\
HPUX_RISC & $\Rightarrow$ VMS_VAX \\
HPUX_RISC & $\Rightarrow$ ULTRIX_RISC
\end{tabular}

\section{Notes:}

1) The following diagram depicts the different representations of the big and little Endian formats. The diagram is based on a 32-bit data type partitioned into four 8-bit bytes. 
Little-Endian

\begin{tabular}{|c|c|c|c|}
\hline 4 & 3 & 2 & 1 \\
\hline High
\end{tabular}

Big-Endian

\begin{tabular}{|l|l|l|l|}
\hline 1 & 2 & 3 & 4 \\
\hline
\end{tabular}

High
Low

2) The following depicts the different representations of VAX and IEEE floating point formats. The diagram is based on a 32-bit data type using little-Endian format.

IEEE Floating Point Format

\begin{tabular}{|l|c|c|}
\hline S & Exponent & Fraction \\
\hline 31 & 2322 & 0
\end{tabular}

VAX Floating Point Format

\begin{tabular}{|l|l|c|c|}
\hline $\begin{array}{c}\text { Fraction } \\
\text { (low) }\end{array}$ & S & Exponent & $\begin{array}{c}\text { Frac. } \\
\text { (high) }\end{array}$ \\
\hline 31 & 15 & 76 & 76
\end{tabular}

3) The following matrix depicts the different representations used by the different hardware and software architecture's.

\begin{tabular}{||l||c||c||c||c|}
\hline & Little-Endian & Big-Endian & VAX Float & IEEE Float \\
\hline \hline VMS_VAX & $\checkmark$ & & $\checkmark$ & \\
\hline \hline ULTRIX_RISC & $\checkmark$ & & & $\checkmark$ \\
\hline \hline HPUX_RISC & & $\checkmark$ & & $\checkmark$ \\
\hline
\end{tabular}

4) The following table lists and describes the conversion routines. It is assumed that all routines are based on 8-bit bytes. Furthermore, within the float conversion process, the bits devoted to the fraction portion assure "about" seven decimal digits of precision.

\begin{tabular}{|l|l||}
\hline Routine & Description \\
\hline \hline cvt_float32 & Converts an array of float(s) to a particular target format \\
\hline cvt_int32 & Converts an array of int(s) to a particular target format \\
\hline cvt_short16 & Converts an array of short(s) to a particular target format \\
\hline get_native_data_ID & Used internal to determine the native data format of a machine \\
\hline
\end{tabular}


5) Rather than using the integer-based conversion routines, a set of four functions could have been used that were designed for the Internet protocol. However, this would have required both the client and server to call the appropriate conversion routine rather than just the client. The set of four conversion functions are described by the following table.

\begin{tabular}{||l||l|}
\hline Function & Description \\
\hline \hline htonl & Convert host-to-network, long integer \\
\hline htons & Convert host-to-network, short integer \\
\hline ntohl & Convert network-to-host, long integer \\
\hline ntohs & Convert network-to-host, short integer \\
\hline
\end{tabular}

(Implicit in these functions is that a short integer occupies 16 bits and a long integer 32 bits. On those systems that have the same byte ordering as the Internet protocols (big Endian), the four functions are null macros.)

6) The converted values occupy the same storage location as the values to be converted (i.e., the values to be converted is overwritten by the converted values). Care must be taken in using the converted values; otherwise, an underflow, overflow or divide by zero exception may occur. 